\title{
Sparse Markov Decision Processes with Causal Sparse Tsallis Entropy Regularization for Reinforcement Learning
}

\author{
Kyungjae Lee, Sungjoon Choi, and Songhwai Oh
}

\begin{abstract}
In this paper, a sparse Markov decision process (MDP) with novel causal sparse Tsallis entropy regularization is proposed. The proposed policy regularization induces a sparse and multi-modal optimal policy distribution of a sparse MDP. The full mathematical analysis of the proposed sparse MDP is provided. We first analyze the optimality condition of a sparse MDP. Then, we propose a sparse value iteration method which solves a sparse MDP and then prove the convergence and optimality of sparse value iteration using the Banach fixed point theorem. The proposed sparse MDP is compared to soft MDPs which utilize causal entropy regularization. We show that the performance error of a sparse MDP has a constant bound, while the error of a soft MDP increases logarithmically with respect to the number of actions, where this performance error is caused by the introduced regularization term. In experiments, we apply sparse MDPs to reinforcement learning problems. The proposed method outperforms existing methods in terms of the convergence speed and performance.
\end{abstract}

\section{INTRODUCTION}

$\mathbf{M}$ Arkov decision processes (MDPs) have been widely used as a mathematical framework to solve stochastic sequential decision problems, such as autonomous driving [1], path planning [2], and quadrotor control [3]. In general, the goal of an MDP is to find the optimal policy function which maximizes the expected return. The expected return is a performance measure of a policy function and it is often defined as the expected sum of discounted rewards. An MDP is often used to formulate reinforcement learning (RL) [4], which aims to find the optimal policy without the explicit specification of stochasticity of an environment, and inverse reinforcement learning (IRL) [5], whose goal is to search the proper reward function that can explain the behavior of an expert who follows the underlying optimal policy.

While the optimal solution of an MDP is a deterministic policy, it is not desirable to apply an MDP to the problems with multiple optimal actions. In perspective of RL, the knowledge of multiple optimal actions makes it possible to cope with unexpected situations. For example, suppose that an autonomous vehicle has multiple optimal routes to reach a given goal. If a traffic accident occurs at the currently selected optimal route, it is possible to avoid the accident by choosing another safe optimal route without additional computation of a new optimal route. For this reason, it is more desirable to learn all possible optimal actions in terms of robustness of a

K. Lee, S. Choi, and S. Oh are with the Department of Electrical and Computer Engineering and ASRI, Seoul National University, Seoul 08826, Korea (e-mail: \{kyungjae.lee, sungjoon.choi, songhwai.oh\}@ cpslab.snu.ac.kr). policy function. In perspective of IRL, since the experts often make multiple decisions in the same situation, a deterministic policy has a limitation in expressing the expert's behavior. For this reason, it is indispensable to model the policy function of an expert as a multi-modal distribution. These reasons give a rise to the necessity of a multi-modal policy model.

In order to address the issues with a deterministic policy function, a causal entropy regularization method has been utilized [6]-[10]. This is mainly due to the fact that the optimal solution of an MDP with causal entropy regularization becomes a softmax distribution of state-action values $Q(s, a)$, i.e., $\pi(a \mid s)=\frac{\exp (Q(s, a))}{\sum^{\prime} \exp \left(Q\left(s, a^{\prime}\right)\right)}$, which is often referred to as a soft MDP [11]. While a softmax distribution has been widely used to model a stochastic policy, it has a weakness in modeling a policy function when the number of actions is large. In other words, the policy function modeled by a softmax distribution is prone to assign non-negligible probability mass to non-optimal actions even if state-action values of these actions are dismissible. This tendency gets worse as the number of actions increases as demonstrated in Figure 1

In this paper, we propose a sparse MDP by presenting a novel causal sparse Tsallis entropy regularization method, which can be interpreted as a special case of Tsallis generalized entropy [12]. The proposed regularization method has a unique property in that the resulting policy distribution becomes a sparse distribution. In other words, the supporting action set which has a non-zero probability mass contains a sparse subset of the action space.

We provide a full mathematical analysis about the proposed sparse MDP. We first derive the optimality condition of a sparse MDP, which is named as a sparse Bellman equation. We show that the sparse Bellman equation is an approximation of the original Bellman equation. Interestingly, we further find the connection between the optimality condition of a sparse MDP and the probability simplex projection problem [13]. We present a sparse value iteration method for solving a sparse MDP problem, where the optimality and convergence are proven using the Banach fixed point theorem [14]. We further analyze the performance gaps of the expected return of the optimal policies obtained by a sparse MDP and a soft MDP compared to that of the original MDP. In particular, we prove that the performance gap between the proposed sparse MDP and the original MDP has a constant bound as the number of actions increases, whereas the performance gap between a soft MDP and the original MDP grows logarithmically. From this property, sparse MDPs have benefits over soft MDPs when 
it comes to solving problems in robotics with a continuous action space.

To validate effectiveness of a sparse MDP, we apply the proposed method to the exploration strategy and the update rule of Q-learning and compare to the $\epsilon$-greedy method and softmax policy [9]. The proposed method is also compared to the deep deterministic policy gradient (DDPG) method [15], which is designed to operate in a continuous action space without discretization. The proposed method shows the state of the art performance compared to other methods as the discretization level of an action space increases.

\section{BACKGROUND}

\section{A. Markov Decision Processes}

A Markov decision process (MDP) has been widely used to formulate a sequential decision making problem. An MDP can be characterized by a tuple $\mathbf{M}=\{\mathcal{S}, \mathcal{F}, \mathcal{A}, d, T, \gamma, \mathbf{r}\}$, where $\mathcal{S}$ is the state space, $\mathcal{F}$ is the corresponding feature space, $\mathcal{A}$ is the action space, $d(s)$ is the distribution of an initial state, $T\left(s^{\prime} \mid s, a\right)$ is the transition probability from $s \in \mathcal{S}$ to $s^{\prime} \in \mathcal{S}$ by taking $a \in \mathcal{A}, \gamma \in(0,1)$ is a discount factor, and $\mathbf{r}$ is the reward function. The objective of an MDP is to find a policy which maximize $\mathbb{E}\left[\sum_{t=0}^{\infty} \gamma^{t} r\left(s_{t}, a_{t}\right) \mid \pi, d, T\right]$, where policy $\pi$ is a mapping from the state space to the action space. For notational simplicity, we denote the expectation of a discounted summation of function $f(s, a)$, i.e., $\mathbb{E}\left[\sum_{t=0}^{\infty} \gamma^{t} f\left(s_{t}, a_{t}\right) \mid \pi, d, T\right]$, by $\mathbb{E}_{\pi}[f(s, a)]$, where $f(s, a)$ is a function of state and action, such as a reward function $\mathbf{r}(s, a)$ or an indicator function $\mathbb{1}_{\left\{s=s^{\prime}\right\}}$. We also denote the expectation of a discounted summation of function $f(s, a)$ conditioned on the initial state, i.e., $\mathbb{E}\left[\sum_{t=0}^{\infty} \gamma^{t} f\left(s_{t}, a_{t}\right) \mid \pi, s_{0}=s, T\right]$, by $\mathbb{E}_{\pi}\left[f(s, a) \mid s_{0}=s\right]$. Finding an optimal policy for an MDP can be formulated as follows:

$$
\begin{array}{ll}
\underset{\pi}{\operatorname{maximize}} & \mathbb{E}_{\pi}\left[\mathbf{r}\left(s_{t}, a_{t}\right)\right] \\
\text { subject to } & \forall s \sum_{a^{\prime}} \pi\left(a^{\prime} \mid s\right)=1, \quad \forall s, a \quad \pi\left(a^{\prime} \mid s\right) \geq 0 .
\end{array}
$$

The necessary condition for the optimal solution of (1) is called the Bellman equation. The Bellman equation is derived from the Bellman's optimality principal as follows:

$$
\begin{aligned}
Q_{\pi}(s, a) & =\mathbf{r}(s, a)+\gamma \sum_{s^{\prime}} V_{\pi}\left(s^{\prime}\right) T\left(s^{\prime} \mid s, a\right) \\
V_{\pi}(s) & =\max _{a^{\prime}} Q\left(s, a^{\prime}\right) \\
\pi(s) & =\arg \max _{a^{\prime}} Q\left(s, a^{\prime}\right),
\end{aligned}
$$

where $V_{\pi}(s)$ is a value function of $\pi$, which is the expected sum of discounted rewards when the initial state is given as $s$, and $Q_{\pi}(s, a)$ is a state-action value function of $\pi$, which is the expected sum of discounted rewards when the initial state and action are given as $s$ and $a$, respectively. Note that the optimal solution is a deterministic function, which is referred to as a deterministic policy.

\section{B. Entropy Regularized Markov Decision Processes}

In order to obtain a multi-modal policy function, an entropyregularized MDP, also known as a soft MDP, has been widely

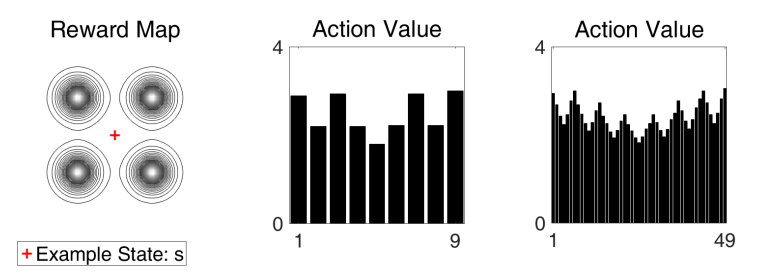

(a) Reward map and action values at state $s$.

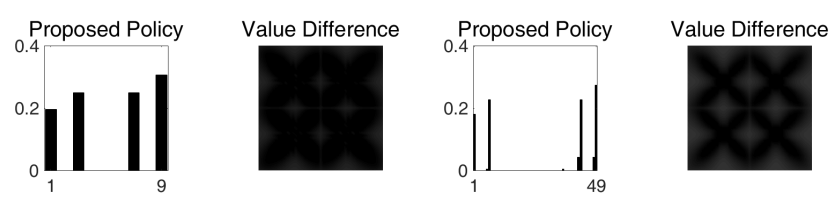

(b) Proposed policy model and value differences (darker is better).

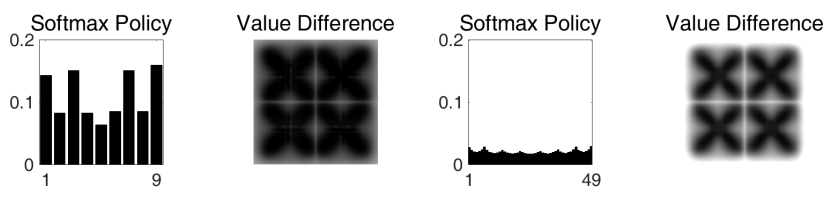

(c) Softmax policy model and value differences (darker is better).

Fig. 1: A 2-dimensional multi-objective environment with point mass dynamics. The state is a location and the action is a velocity bounded with $[-3,3] \times[-3,3]$. (a) The left figure shows the reward map with four maxima (multiple objectives). The action space is discretized into two levels: 9 (low resolution) and 49 (high resolution). The middle (resp., right) figure shows the optimal action value at state $s$ indicated as red cross point when the number of action is 9 (resp., 49). (b) The first and third figure indicate the proposed policy distributions at state $s$ induced by the action values in (a). The second and fourth figure show a map of the performance difference between the proposed policy and the optimal policy at each state when the number of action is 9 and 49, respectively. The larger the error, the brighter the color of the state. (c) All figures are obtained in the same way as (b) by replacing the proposed policy with a softmax policy. This example shows that the proposed policy model is less affected when the number of actions increases.

used [8]-[11]. In a soft MDP, causal entropy regularization over $\pi$ is introduced to obtain a multi-modal policy distribution, i.e., $\pi(a \mid s)$. Since causal entropy regularization penalizes a deterministic distribution, it makes an optimal policy of a soft MDP to be a softmax distribution. A soft MDP is formulated as follows:

$$
\begin{array}{ll}
\underset{\pi}{\operatorname{maximize}} & \mathbb{E}_{\pi}\left[\mathbf{r}\left(s_{t}, a_{t}\right)\right]+\alpha H(\pi) \\
\text { subject to } & \forall s \sum_{a^{\prime}} \pi\left(a^{\prime} \mid s\right)=1, \quad \forall s, a \quad \pi\left(a^{\prime} \mid s\right) \geq 0,
\end{array}
$$

where $H(\pi) \triangleq \mathbb{E}_{\pi}\left[-\log \left(\pi\left(a_{t} \mid s_{t}\right)\right)\right]$ is a $\gamma$-discounted causal entropy and $\alpha$ is a regularization coefficient. This problem (2) has been extensively studied in [6], [8], [11]. In [11], a 
soft Bellman equation and the optimal policy distribution are derived from the Karush Kuhn Tucker (KKT) conditions as follows:

$$
\begin{aligned}
Q_{\pi}^{\text {soft }}(s, a) & =r(s, a)+\gamma \sum_{s^{\prime}} V_{\pi}^{\text {soft }}\left(s^{\prime}\right) T\left(s^{\prime} \mid s, a\right) \\
V_{\pi}^{\text {soft }}(s) & =\alpha \log \left(\sum_{a^{\prime}} \exp \left(\frac{Q_{\pi}^{\text {soft }}\left(s, a^{\prime}\right)}{\alpha}\right)\right) \\
\pi(a \mid s) & =\frac{\exp \left(\frac{Q_{\pi}^{\text {soft }}(s, a)}{\alpha}\right)}{\sum_{a^{\prime}} \exp \left(\frac{Q_{\pi}^{\text {soft }}\left(s, a^{\prime}\right)}{\alpha}\right)}
\end{aligned}
$$

where

$$
\begin{aligned}
V_{\pi}^{\text {soft }}(s) & =\mathbb{E}_{\pi}\left[r\left(s_{t}, a_{t}\right)-\alpha \log \left(\pi\left(a_{t} \mid s_{t}\right)\right) \mid s_{0}=s\right] \\
Q_{\pi}^{\text {soft }}(s, a) & =\mathbb{E}_{\pi}\left[r\left(s_{t}, a_{t}\right)-\alpha \log \left(\pi\left(a_{t} \mid s_{t}\right)\right) \mid s_{0}=s, a_{0}=a\right] .
\end{aligned}
$$

$V_{\pi}^{\text {soft }}(s)$ is a soft value of $\pi$ indicating the expected sum of rewards including the entropy of a policy, obtained by starting at state $s$ and $Q_{\pi}^{\text {soft }}(s, a)$ is a soft state-action value of $\pi$, which is the expected sum of rewards obtained by starting at state $s$ by taking action $a$. Note that the optimal policy distribution is a softmax distribution. In [11], a soft value iteration method is also proposed and the optimality of soft value iteration is proved. By using causal entropy regularization, the optimal policy distribution of a soft MDP is able to represent a multi-modal distribution.

The causal entropy regularization has an effect of making the resulting policy of a soft MDP closer to a uniform distribution as the number of actions increases. To handle this issue, we propose a novel regularization method whose resulting policy distribution still has multiple modes (a stochastic policy) but the performance loss is less than a softmax policy distribution.

\section{Sparse Markov Decision Processes}

We propose a sparse Markov decision process by introducing a novel causal sparse Tsallis entropy regularizer:

$$
\begin{aligned}
W(\pi) & \triangleq \mathbb{E}\left[\sum_{t=0}^{\infty} \gamma^{t} \frac{1}{2}\left(1-\pi\left(a_{t} \mid s_{t}\right)\right) \mid \pi, d, T\right] \\
& =\mathbb{E}_{\pi}\left[\frac{1}{2}(1-\pi(a \mid s))\right] .
\end{aligned}
$$

By adding $W(\pi)$ to the objective function of 1 , we aim to solve the following optimization problem:

$$
\begin{array}{ll}
\underset{\pi}{\operatorname{maximize}} & \mathbb{E}_{\pi}[\mathbf{r}(s, a)]+\alpha W(\pi) \\
\text { subject to } & \forall s \sum_{a^{\prime}} \pi\left(a^{\prime} \mid s\right)=1, \forall s, a \pi\left(a^{\prime} \mid s\right) \geq 0,
\end{array}
$$

where $\alpha>0$ is a regularization coefficient. We will first derive the sparse Bellman equation from the necessary condition of (3). Then by observing the connection between the sparse Bellman equation and the probability simplex projection, we show that the optimal policy becomes a sparsemax distribution, where the sparsity can be controlled by $\alpha$. In addition, we present a sparse value iteration algorithm where the optimality is guaranteed using the Banach's fixed point theorem. The detailed derivations of lemmas and theorems in this paper can be found in Appendix A

\section{A. Notations and Properties}

We first introduce notations and properties used in the paper. In Table I] all notations and definitions are summarized. The utility, value, state visitation can be compactly expressed as below in terms of vectors and matrices:

$$
\begin{array}{rlrl}
J_{\pi}^{s p} & =d^{\top} G_{\pi}^{-1} r_{\pi}^{s p}, & V_{\pi}^{s p} & =G_{\pi}^{-1} r_{\pi}^{s p} \\
J_{\pi}^{s o f t} & =d^{\top} G_{\pi}^{-1} r_{\pi}^{s o f t}, \quad V_{\pi}^{s o f t} & =G_{\pi}^{-1} r_{\pi}^{s o f t}, \quad \rho_{\pi}=d^{\top} G_{\pi}^{-1}
\end{array}
$$

where $x^{\top}$ is the transpose of vector $x, G_{\pi}=\left(I-\gamma T_{\pi}\right)$, sp indicates a sparse MDP problem and soft indicates a soft MDP problem.

\section{B. Sparse Bellman Equation from Karush-Kuhn-Tucker con- ditions}

The sparse Bellman equation can be derived from the necessary conditions of an optimal solution of a sparse MDP. We carefully investigate the Karush Kuhn Tucker (KKT) conditions, which indicate necessary conditions for a solution to be optimal when some regularity conditions about the feasible set are satisfied. The feasible set of a sparse MDP satisfies linearity constraint qualification [16] since the feasible set consists of linear afine functions. In this regards, the optimal solution of a sparse MDP necessarily satisfy KKT conditions as follows.

Theorem 1. If a policy distribution $\pi$ is the optimal solution of a sparse MDP (3), then $\pi$ and the corresponding sparse value function $V_{\pi}^{s p}$ necessarily satisfy following equations for all state and action pairs:

$$
\begin{aligned}
Q_{\pi}^{s p}(s, a) & =\mathbf{r}(s, a)+\gamma \sum_{s^{\prime}} V_{\pi}^{s p}\left(s^{\prime}\right) T\left(s^{\prime} \mid s, a\right) \\
V_{\pi}^{s p}(s) & =\alpha\left[\frac{1}{2} \sum_{a \in S(s)}\left(\left(\frac{Q_{\pi}^{s p}(s, a)}{\alpha}\right)^{2}-\tau\left(\frac{Q_{\pi}^{s p}(s, \cdot)}{\alpha}\right)^{2}\right)+\frac{1}{2}\right] \\
\pi(a \mid s) & =\max \left(\frac{Q_{\pi}^{s p}(s, a)}{\alpha}-\tau\left(\frac{Q_{\pi}^{s p}(s, \cdot)}{\alpha}\right), 0\right),
\end{aligned}
$$

where $\tau\left(\frac{Q_{\pi}^{s p}(s, \cdot)}{\alpha}\right)=\frac{\sum_{a \in S(s)} \frac{Q_{\pi}^{s p}(s, a)}{\alpha}-1}{K_{s}}, S(s)$ is a set of actions satisfying $1+i \frac{Q_{\pi}^{s p}\left(s, a_{(i)}\right)}{\alpha}>\sum_{j=0}^{i} \frac{Q_{\pi}^{s p}\left(s, a_{(j)}\right)}{\alpha}$ with $a_{(i)}$ indicating the action with the $i$ th largest action value $Q_{\pi}^{s p}\left(s, a_{(i)}\right)$, and $K_{s}$ is the cardinality of $S(s)$.

The full proof of Theorem 1 is provided in Appendix A-A The proof depends on the KKT condition where the derivative of a Lagrangian objective function with respect to policy $\pi(a \mid s)$ becomes zero at the optimal solution, the stationary condition. From (4), it can be shown that the optimal solution obtained from the sparse MDP assigns zero probability to the action whose action value $Q^{s p}(s, a)$ is below the threshold $\tau\left(\frac{Q_{\pi}^{s p}(s, \cdot)}{\alpha}\right)$ and the optimal policy assigns positive probability to near optimal actions in proportion to their action values, where the threshold $\tau\left(\frac{Q_{\pi}^{s p}(s, \cdot)}{\alpha}\right)$ determines the range of near optimal actions. This property makes the optimal policy to have a sparse distribution and prevents the performance drop caused by assigning non-negligible positive probabilities to non-optimal actions, which often occurs in a soft MDP.

From the definitions of $S(s)$ and $\pi(a \mid s)$, we can further observe an interesting connection between the sparse Bellman equation and the probability simplex projection problem [13]. 


\begin{tabular}{|c|c|c|}
\hline Terms & sparse MDP & soft MDP \\
\hline Utility & $\begin{array}{l}J_{\pi}^{s p} \triangleq \mathbb{E}_{\pi}\left[\mathbf{r}\left(s^{\prime}, a^{\prime}\right)+\frac{\alpha}{2}\left(1-\pi\left(a^{\prime} \mid s^{\prime}\right)\right)\right] \\
=\sum_{s} d(s) V_{\pi}^{s p}(s)=\sum_{s} \mathbf{r}_{\pi}^{s p}(s) \rho_{\pi}(s)\end{array}$ & $\begin{array}{l}J_{\pi}^{\text {soft }} \triangleq \mathbb{E}_{\pi}\left[\mathbf{r}\left(s^{\prime}, a^{\prime}\right)-\alpha \log \left(\pi\left(a^{\prime} \mid s^{\prime}\right)\right)\right] \\
=\sum_{s} d(s) V_{\pi}^{\text {soft }}(s)=\sum_{s} \mathbf{r}_{\pi}^{\text {soft }}(s) \rho_{\pi}(s)\end{array}$ \\
\hline Value & $\begin{array}{l}V_{\pi}^{s p}(s) \\
\triangleq \mathbb{E}_{\pi}\left[\mathbf{r}\left(s^{\prime}, a^{\prime}\right)+\frac{\alpha}{2}\left(1-\pi\left(a^{\prime} \mid s^{\prime}\right)\right) \mid s_{0}=s\right] \\
=\mathbf{r}_{\pi}^{s p}(s)+\gamma \sum_{s^{\prime}} V_{\pi}^{s p}\left(s^{\prime}\right) T_{\pi}\left(s^{\prime} \mid s\right)\end{array}$ & $\begin{array}{l}V_{\pi}^{\text {soft }}(s) \\
\triangleq \mathbb{E}_{\pi}\left[\mathbf{r}\left(s^{\prime}, a^{\prime}\right)-\alpha \log \left(\pi\left(a^{\prime} \mid s^{\prime}\right)\right) \mid s_{0}=s\right] \\
=\mathbf{r}_{\pi}^{\text {soft }}(s)+\gamma \sum_{s^{\prime}} V_{\pi}^{\text {soft }}\left(s^{\prime}\right) T_{\pi}\left(s^{\prime} \mid s\right)\end{array}$ \\
\hline Action value & $\begin{array}{l}Q_{\pi}^{s p}(s, a) \triangleq \\
\mathbf{r}(s, a)+\gamma \sum_{s^{\prime}} V_{\pi}^{s p}\left(s^{\prime}\right) T\left(s^{\prime} \mid s, a\right)\end{array}$ & $\begin{array}{l}Q_{\pi}^{s o f t}(s, a) \triangleq \\
\mathbf{r}(s, a)+\gamma \sum_{s^{\prime}} V_{\pi}^{\text {soft }}\left(s^{\prime}\right) T\left(s^{\prime} \mid s, a\right)\end{array}$ \\
\hline $\begin{array}{l}\text { Expected } \\
\text { State Reward }\end{array}$ & $\begin{array}{l}\mathbf{r}_{\pi}^{s p}(s) \triangleq \\
\sum_{a^{\prime}}\left(\mathbf{r}\left(s, a^{\prime}\right)+\frac{\alpha}{2}\left(1-\pi\left(a^{\prime} \mid s\right)\right)\right) \pi\left(a^{\prime} \mid s\right)\end{array}$ & $\begin{array}{l}\mathbf{r}_{\pi}^{\text {soft }}(s) \triangleq \\
\sum_{a^{\prime}}\left(\mathbf{r}\left(s, a^{\prime}\right)-\alpha \log \left(\pi\left(a^{\prime} \mid s\right)\right)\right) \pi\left(a^{\prime} \mid s\right)\end{array}$ \\
\hline $\begin{array}{l}\text { Policy Regu- } \\
\text { larization }\end{array}$ & $\begin{array}{l}W(\pi) \triangleq \mathbb{E}_{\pi}\left[\frac{1}{2}(1-\pi(a \mid s))\right] \\
=\sum_{s, a} \frac{1}{2}(1-\pi(a \mid s)) \pi(a \mid s) \rho(s)\end{array}$ & $\begin{array}{l}H(\pi)=\mathbb{E}_{\pi}[-\pi(a \mid s) \log (\pi(a \mid s))] \\
=\sum_{s, a}-\pi(a \mid s) \log (\pi(a \mid s)) \rho(s)\end{array}$ \\
\hline $\begin{array}{l}\text { Max Approxi- } \\
\text { mation }\end{array}$ & $\operatorname{spmax}(z) \triangleq \frac{1}{2} \sum_{i=1}^{K}\left(z_{(i)}^{2}-\tau(z)^{2}\right)+\frac{1}{2}$ & $\operatorname{logsumexp}(z) \triangleq \log \sum_{i} \exp \left(z_{i}\right)$ \\
\hline $\begin{array}{l}\text { Value } \\
\text { Iteration } \\
\text { Operator }\end{array}$ & $U^{s p}(x)(s)=\alpha \operatorname{spmax}\left(\frac{r(s, \cdot)+\gamma \sum_{s^{\prime}} x\left(s^{\prime}\right) T\left(s^{\prime} \mid s, \cdot\right)}{\alpha}\right)$ & $U^{\text {soft }}(x)(s)=\alpha \operatorname{logsumexp}\left(\frac{r(s, \cdot)+\gamma \sum_{s^{\prime}} x\left(s^{\prime}\right) T\left(s^{\prime} \mid s, \cdot\right)}{\alpha}\right)$ \\
\hline $\begin{array}{l}\text { State } \\
\text { Visitation }\end{array}$ & \multicolumn{2}{|c|}{$\rho_{\pi}(s) \triangleq \mathbb{E}_{\pi}\left[\mathbb{1}_{\left\{s^{\prime}=s\right\}}\right]=d(s)+\gamma \sum_{s^{\prime}, a^{\prime}} T\left(s \mid s^{\prime}, a^{\prime}\right) \rho_{\pi}\left(s^{\prime}, a^{\prime}\right)$} \\
\hline $\begin{array}{l}\text { State Action } \\
\text { Visitation }\end{array}$ & \multicolumn{2}{|c|}{$\rho_{\pi}(s, a) \triangleq \mathbb{E}_{\pi}\left[\mathbb{1}_{\left\{s^{\prime}=s, a^{\prime}=a\right\}}\right]=\pi(a \mid s) d(s)+\gamma \sum_{s^{\prime}, a^{\prime}} \pi(a \mid s) T\left(s \mid s^{\prime}, a^{\prime}\right) \rho_{\pi}\left(s^{\prime}, a^{\prime}\right)$} \\
\hline $\begin{array}{l}\text { Transition } \\
\text { Probability } \\
\text { given } \pi\end{array}$ & \multicolumn{2}{|c|}{$T_{\pi}\left(s^{\prime} \mid s\right) \triangleq \sum_{a} T\left(s^{\prime} \mid s, a\right) \pi(a \mid s)$} \\
\hline
\end{tabular}

TABLE I: Notations and Properties

\section{Probability Simplex Projection and SparseMax Operation}

The probability simplex projection [13] is a well known problem of projecting a $d$-dimensional vector into a $d-1$ dimensional probability simplex in an Euclidean metric sense. A probability simplex projection problem is defined as follows:

$$
\begin{array}{ll}
\underset{p}{\operatorname{minimize}} & \frac{1}{2}\|p-z\|_{2}^{2} \\
\text { subject to } & \sum_{i=1}^{d} p_{i}=1, \quad p_{i} \geq 0, \forall i=1, \cdots, d,
\end{array}
$$

where $z$ is a given $d$-dimensional vector, $d$ is the dimension of $p$ and $z$, and $p_{i}$ is the $i$ th element of $p$. Let $z_{(i)}$ be the $i$ th largest element of $z$ and $\operatorname{supp}(z)$ be the supporting set of the optimal solution as defined by $\operatorname{supp}(z)=\left\{z_{(i)} \mid 1+i z_{(i)}>\right.$ $\left.\sum_{j=1}^{i} z_{(j)}\right\}$. It is a well known fact that the problem $\sqrt{5}$ has a closed form solution which is $p_{i}^{*}(z)=\max \left(z_{i}-\tau(z), 0\right)$, where $i$ indicates the $i$ th dimension, $p_{i}^{*}(z)$ is the $i$ th element of the optimal solution for fixed $z$, and $\tau(z)=\frac{\sum_{i=1}^{K} z_{(i)}-1}{K}$ with $K=|\operatorname{supp}(z)|$ [13], [17].

Interestingly, the optimal solution $p^{*}(\cdot), \tau(\cdot)$ and the supporting set $\operatorname{supp}(\cdot)$ of (5) can be precisely matched to those of the sparse Bellman equation (4). From this observation, it can be shown that the optimal policy distribution of a sparse MDP is the projection of $Q_{\pi}^{s p}(s, \cdot)$ into a probability simplex. Note that we refer $p^{*}(\cdot)$ as a sparsemax distribution.

More surprisingly, $V_{\pi}^{s p}$ can be represented as an approximation of the max operation derived from $p^{*}(z)$. A differentiable approximation of the max operation is defined as follows:

$$
\operatorname{spmax}(z) \triangleq \frac{1}{2} \sum_{i=1}^{K}\left(z_{(i)}^{2}-\tau(z)^{2}\right)+\frac{1}{2}
$$

We call $\operatorname{spmax}(z)$ as sparsemax. In [17], it is proven that $\operatorname{spmax}(z)$ is an indefinite integral of $p^{*}(z)$, i.e., $\operatorname{spmax}(z)=$ $\int\left(p^{*}(z)\right)^{\top} \mathbf{d} z+C$, where $C$ is a constant and, in our case, $C=$ $\frac{1}{2}$. We provide simple upper and lower bounds of $\operatorname{spmax}(z)$ with respect to $\max (z)$

$$
\max (z) \leq \alpha \operatorname{spmax}\left(\frac{z}{\alpha}\right) \leq \max (z)+\alpha \frac{d-1}{2 d} .
$$

The lower bound of sparsemax is shown in [17]. However, we provide another proof of the lower bound and the proof for the upper bound in Appendix A-B.

The bounds (7) show that sparsemax is a bounded and smooth approximation of max and, from this fact, (4) can be interpreted as an approximation of the original Bellman equation. Using this notation, $V_{\pi}^{s p}$ can be rewritten as,

$$
V_{\pi}^{s p}(s)=\alpha \operatorname{spmax}\left(\frac{Q_{\pi}^{s p}(s, \cdot)}{\alpha}\right) .
$$

\section{Supporting Set of Sparse Optimal Policy}

The supporting set $S(s)$ of a sparse MDP is a set of actions with nonzero probabilities and the cardinality of $S(s)$ can be controlled by regularization coefficient $\alpha$, while the supporting set of a soft MDP is always the same as the entire action space. In a sparse MDP, actions assigned with non-zero probability must satisfy the following inequality:

$$
\alpha+i Q_{\pi}^{s p}\left(s, a_{(i)}\right)>\sum_{j=1}^{i} Q_{\pi}^{s p}\left(s, a_{(j)}\right),
$$

where $a_{(i)}$ indicates the action with the $i$ th largest action value. From this inequality, it can be shown that $\alpha$ controls the margin between the largest action value and the others included in the supporting set. In other words, as $\alpha$ increases, 
the cardinality of a supporting set increases since the action values that satisfy (8) increase. Conversely, as $\alpha$ decreases, the supporting set decreases. In extreme cases, if $\alpha$ goes zero, only $a_{(1)}$ will be included in $S(s)$ and if $\alpha$ goes infinity, the entire actions will be included in $S(s)$. On the other hand, in a soft MDP, the supporting set of a softmax distribution cannot be controlled by the regularization coefficient $\alpha$ even if the sharpness of the softmax distribution can be adjusted. This property makes sparse MDPs have an advantage over soft MDPs, since we can give a zero probability to non-optimal actions by controlling $\alpha$.

\section{E. Connection to Tsallis Generalized Entropy}

The notion of the Tsallis entropy was introduced by $\mathrm{C}$. Tsallis as a general extension of entropy [12] and the Tsallis entropy has been widely used to describe thermodynamic systems and molecular motions. Surprisingly, the proposed regularization is closely related to a special case of the Tsallis entropy. The Tsallis entropy is defined as follows:

$$
S_{q, k}(p)=\frac{k}{q-1}\left(1-\sum_{i} p_{i}^{q}\right)
$$

where $p$ is a probability mass function, $q$ is a parameter called entropic-index, and $k$ is a positive real constant. Note that, if $q \rightarrow 1$ and $k=1, S_{1,1}(p)$ is the same as entropy, i.e., $-\sum_{i} p_{i} \log \left(p_{i}\right)$. In [11], [18], it is shown that $H(\pi)$ is an extension of $S_{1,1}(\pi(\cdot \mid s))$ since $H(\pi)=\mathbb{E}_{\pi}\left[S_{(1,1)}(\pi(\cdot \mid s))\right]=$ $\sum_{s, a}-\pi(a \mid s) \log (\pi(a \mid s)) \rho(s)$.

We discover the connection between the Tsallis entropy and the proposed regularization when $q=2$ and $k=\frac{1}{2}$.

Theorem 2. The proposed policy regularization $W(\pi)$ is an extension of the Tsallis entropy with parameters $q=2$ and $k=\frac{1}{2}$ to the version of causal entropy, i.e.,

$$
W(\pi)=\mathbb{E}_{\pi}\left[S_{2, \frac{1}{2}}(\pi(\cdot \mid s))\right] .
$$

The proof is provided in Appendix A-D

From this theorem, $W(\pi)$ can be interpreted as an extension of $S_{2, \frac{1}{2}}(p)$ to the case of causally conditioned distribution, similarly to the causal entropy.

\section{Sparse VAlue Iteration}

In this section, we propose an algorithm for solving a causal sparse Tsallis entropy regularized MDP problem. Similar to the original MDP and a soft MDP, the sparse version of value iteration can be induced from the sparse Bellman equation. We first define a sparse Bellman operation $U^{s p}: \mathbb{R}^{|\mathcal{S}|} \rightarrow \mathbb{R}^{|\mathcal{S}|}$ : for all $s$,

$U^{s p}(x)(s)=\alpha \operatorname{spmax}\left(\frac{r(s, \cdot)+\gamma \sum_{s^{\prime}} x\left(s^{\prime}\right) T\left(s^{\prime} \mid s, \cdot\right)}{\alpha}\right)$,

where $x$ is a vector in $\mathbb{R}^{|\mathcal{S}|}$ and $U^{s p}(x)$ is the resulting vector after applying $U^{s p}$ to $x$ and $U^{s p}(x)(s)$ is the element for state $s$ in $U^{s p}(x)$. Then, the sparse value iteration algorithm can be described simply as

$$
x_{i+1}=U^{s p}\left(x_{i}\right),
$$

where $i$ is the number of iterations. In the following section, we show the convergence and the optimality of the proposed sparse value iteration method.

\section{A. Optimality of Sparse Value Iteration}

In this section, we prove the convergence and optimality of the sparse value iteration method. We first show that $U^{s p}$ has monotonic and discounting properties and, by using those properties, we prove that $U^{s p}$ is a contraction. Then, by the Banach fixed point theorem, repeatedly applying $U^{s p}$ for an arbitrary initial point always converges into the unique fixed point.

Lemma 1. $U^{s p}$ is monotone: for $x, y \in \mathbb{R}^{|\mathcal{S}|}$, if $x \leq y$, then $U^{s p}(x) \leq U^{s p}(y)$, where $\leq$ indicates an element-wise inequality.

Lemma 2. For any constant $c \in \mathbb{R}, U^{s p}(x+c \mathbf{1})=U^{s p}(x)+$ $\gamma c 1$, where $1 \in \mathbb{R}^{|\mathcal{S}|}$ is a vector of all ones.

The full proofs can be found in Appendix A-E The proofs of Lemma 1 and Lemma 2 rely on the bounded property of the sparsemax operation. It is possible to prove that the sparse Bellman operator $U^{s p}$ is a contraction using Lemma 1 and Lemma 2 as follows:

Lemma 3. $U^{s p}$ is a $\gamma$-contraction mapping and have a unique fixed point, where $\gamma$ is in $(0,1)$ by definition.

Using Lemma 1, Lemma 2, and Lemma 3, the optimality and convergence of sparse value iteration can be proven.

Theorem 3. Sparse value iteration converges to the optimal value of (3).

The proof can be found in Appendix A-E Theorem 3 is proven using the uniqueness of the fixed point of $U^{s p}$ and the sparse Bellman equation.

\section{Performance ERror Bounds for Sparse VAlue ITERATION}

We prove the bounds of the performance gap between the policy obtained by a sparse MDP and the policy obtained by the original MDP, where this performance error is caused by regularization. The boundedness of (7) plays an crucial role to prove the error bounds. The performance bounds can be derived from bounds of sparsemax. A similar approach can be applied to prove the error bounds of a soft MDP since a log-sum-exp function is also a bounded approximation of the max operation. Comparison of log-sum-exp and sparsemax operation is provided in Appendix $\mathrm{A}-\mathrm{C}$

Before explaining the performance error bounds, we introduce two useful propositions which are employed to prove the performance error bounds of a sparse MDP and a soft MDP. We first prove an important fact which shows that the optimal values of sparse value iteration and soft value iteration are greater than that of the original MDP.

Lemma 4. Let $U$ and $U^{\text {soft }}$ be the Bellman operations of an original MDP and soft MDP, respectively, such that, for state 


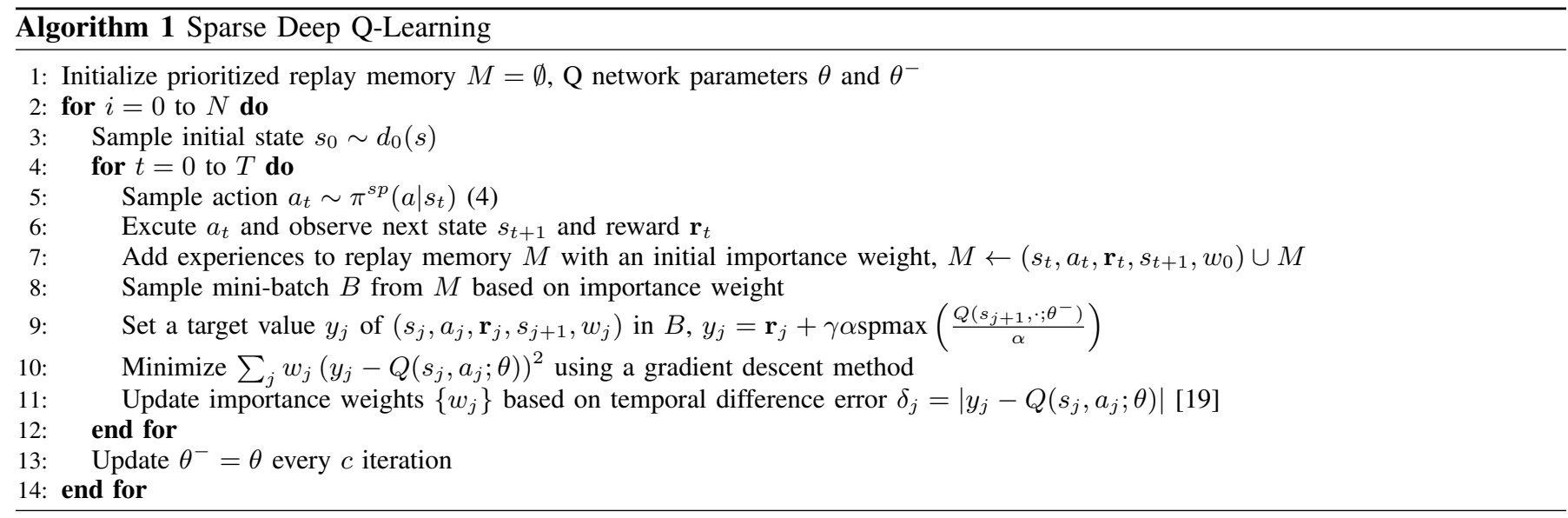

$s$ and $x \in \mathbb{R}^{|\mathcal{S}|}$,

$U(x)(s)=\max _{a^{\prime}}\left(r\left(s, a^{\prime}\right)+\gamma \sum_{s^{\prime}} x\left(s^{\prime}\right) T\left(s^{\prime} \mid s, a^{\prime}\right)\right)$

$U^{s o f t}(x)(s)=\alpha \log \sum_{a^{\prime}} \exp \left(\frac{r\left(s, a^{\prime}\right)+\gamma \sum_{s^{\prime}} x\left(s^{\prime}\right) T\left(s^{\prime} \mid s, a^{\prime}\right)}{\alpha}\right)$.

Then following inequalities hold for every integer $n>0$ :

$$
U^{n}(x) \leq\left(U^{s p}\right)^{n}(x), \quad U^{n}(x) \leq\left(U^{\text {soft }}\right)^{n}(x),
$$

where $U^{n}$ (resp., $\left(U^{s p}\right)^{n}$ ) is the result after applying $U$ (resp., $\left.U^{s p}\right) n$ times. In addition, let $x_{*}, x_{*}^{s p}$ and $x_{*}^{s o f t}$ be the fixed points of $U, U^{s p}$ and $U^{s o f t}$, respectively. Then, following inequalities also hold:

$$
x_{*} \leq x_{*}^{s p}, \quad x_{*} \leq x_{*}^{s o f t} .
$$

The detailed proof is provided in Appendix A-F Lemma 4 shows that the optimal values, $V_{\pi}^{s p}$ and $V_{\pi}^{\text {soft }}$, obtained by sparse value iteration and soft value iteration are always greater than the original optimal value $V_{\pi}$. Intuitively speaking, the reason for this inequality is due to the regularization term, i.e., $W(\pi)$ or $H(\pi)$, added to the objective function.

Now, we discuss other useful properties about the proposed causal sparse Tsallis entropy regularization $W(\pi)$ and causal entropy regularization $H(\pi)$.

Lemma 5. $W(\pi)$ and $H(\pi)$ have following upper bounds:

$$
W(\pi) \leq \frac{1}{1-\gamma} \frac{|\mathcal{A}|-1}{2|\mathcal{A}|}, \quad H(\pi) \leq \frac{\log (|\mathcal{A}|)}{1-\gamma}
$$

where $|\mathcal{A}|$ is the cardinality of the action space $\mathcal{A}$.

The proof is provided in Appendix A-F Theorem 5 can be induced by extending the upper bound of $S_{1,1}(\pi)$ and $S_{2, \frac{1}{2}}(\pi)$ to the causal entropy and causal sparse Tsallis entropy.

By using Lemma 4 and Lemma 5, the performance bounds for a sparse MDP and a soft MDP can be derived as follows.

Theorem 4. Following inequalities hold:

$$
\mathbb{E}_{\pi^{*}}(\mathbf{r}(s, a))-\frac{\alpha}{1-\gamma} \frac{|\mathcal{A}|-1}{2|\mathcal{A}|} \leq \mathbb{E}_{\pi^{s p}}(\mathbf{r}(s, a)) \leq \mathbb{E}_{\pi^{*}}(\mathbf{r}(s, a)),
$$

where $\pi^{*}$ and $\pi^{s p}$ are the optimal policy obtained by the original MDP and a sparse MDP, respectively.
Theorem 5. Following inequalities hold:

$$
\mathbb{E}_{\pi^{*}}(\mathbf{r}(s, a))-\frac{\alpha}{1-\gamma} \log (|\mathcal{A}|) \leq \mathbb{E}_{\pi^{s o f t}}(\mathbf{r}(s, a)) \leq \mathbb{E}_{\pi^{*}}(\mathbf{r}(s, a))
$$

where $\pi^{*}$ and $\pi^{\text {soft }}$ are the optimal policy obtained by the original MDP and a soft MDP, respectively.

The proofs of Theorem 4 and Theorem 5 can be found in Appendix A-F These error bounds show us that the expected return of the optimal policy of a sparse MDP has always tighter error bounds than that of a soft MDP. Moreover, it can be also known that the bounds for the proposed sparse MDP converges to a constant $\frac{\alpha}{2(1-\gamma)}$ as the number of actions increases, whereas the error bounds of soft MDP grows logarithmically.

This property has a clear benefit when a sparse MDP is applied to a robotic problem with a continuous action space. To apply an MDP to a continuous action space, a discretization of the action space is essential and a fine discretization is required to obtain a solution which is closer to the underlying continuous optimal policy. Accordingly, the number of actions becomes larger as the level of discretization increases. In this case, a sparse MDP has advantages over a soft MDP in that the performance error of a sparse MDP is bounded by a constant factor as the number of actions increases, whereas performance error of optimal policy of a soft MDP grows logarithmically.

\section{Sparse Exploration AND Update Rule FOR SPARSE DEEP Q-LEARNING}

In this section, we first propose sparse Q-learning and further extend to sparse deep Q-learning where a sparsemax policy and the sparse Bellman equation are employed as a exploration method and update rule.

Sparse Q-learning is a model free method to solve the proposed sparse MDP without the knowledge of transition probabilities. In other words, when the transition probability $T\left(s^{\prime} \mid a, s\right)$ is unknown but sampling from $T\left(s^{\prime} \mid a, s\right)$ is possible, sparse Q-learning estimates an optimal $Q^{s p}$ of the sparse MDP using sampling, as Q-learning finds an approximated value of an optimal $Q$ of the conventional MDP. Similar to Q-learning, the update equation of sparse Q-learning is derived 


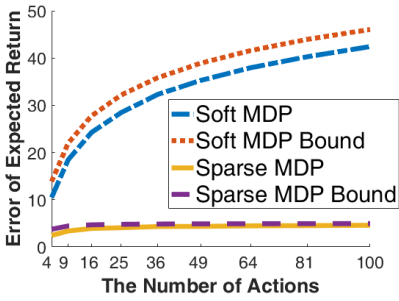

(a) Performance Bounds

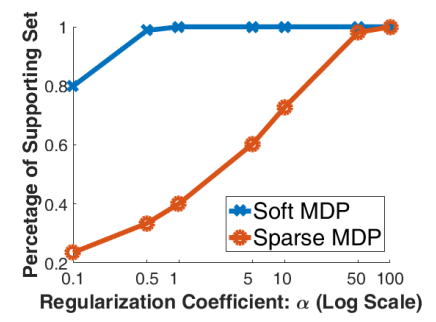

(b) Supporting Set Comparison
Fig. 2: (a) The performance gap is calculated as the absolute value of the difference between the performance of sparse MDP or soft MDP and the performance of an original MDP. (b) The ratio of the number of supporting actions to the total number of actions is shown. The action space of unicycle dynamics is discretized into 25 actions.

from the sparse Bellman equation,

$$
\begin{aligned}
& Q^{s p}\left(s_{i}, a_{i}\right) \leftarrow Q^{s p}\left(s_{i}, a_{i}\right)+ \\
& \eta(i)\left[\mathbf{r}\left(s_{i}, a_{i}\right)+\gamma \alpha \operatorname{spmax}\left(\frac{Q^{s p}\left(s_{i+1}, \cdot\right)}{\alpha}\right)-Q\left(s_{i}, a_{i}\right)\right],
\end{aligned}
$$

where $i$ indicates the number of iterations and $\eta(i)$ is a learning rate. If the learning rate $\eta(i)$ satisfies $\sum_{i=0}^{\infty} \eta(i)=\infty$ and $\sum_{i=0}^{\infty} \eta(i)^{2}<\infty$, then, as the number of samples increases to infinity, sparse Q-learning converges to the optimal solution of a sparse MDP. The proof of the convergence and optimality of sparse Q-learning is the same as that of the standard Qlearning [20].

The proposed sparse Q-learning can be easily extended to sparse deep Q-learning using a deep neural network as an estimator of the sparse $\mathrm{Q}$ value. In each iteration, sparse deep Q-learning performs a gradient descent step to minimize the squared loss $(y-Q(s, a ; \theta))^{2}$, where $\theta$ is the parameter of the $\mathrm{Q}$ network. Here, $y$ is the target value defined as follows:

$$
y=\mathbf{r}(s, a)+\gamma \alpha \operatorname{spmax}\left(\frac{Q\left(s^{\prime}, \cdot ; \theta\right)}{\alpha}\right),
$$

where $s^{\prime}$ is the next state sampled by taking action $a$ at the state $s$ and $\theta$ indicates network parameters.

Moreover, we employ the sparsemax policy as the exploration strategy where the policy distribution is computed by (4) with action values estimated by a deep Q network. The sparsemax policy excludes the action whose estimated action value is too low to be re-explored, by assigning zero probability mass. The effectiveness of the sparsemax exploration is investigated in Section VII

For stable convergence of a $\mathrm{Q}$ network, we utilize double Qlearning [21], where the parameter $\theta$ for obtaining a policy and the parameter $\theta^{-}$for computing the target value are separated and $\theta^{-}$is updated to $\theta$ at every predetermined iterations. In other words, double Q-learning prevents instability of deep Q-learning by slowly updating the target value. Prioritized experience replay [19] is also applied where the optimization of a network proceeds in consideration of the importance of experience. The whole process of sparse deep Q-learning is summarized in Algorithm 1

\section{EXPERIMENTS}

We first verify Theorem 4. Theorem 5 and the effect of (8) in simulation. For verification of Theorem 4 and Theorem 5 . we measure the performance of the expected return while increasing the number of actions, $|\mathcal{A}|$. For verification of the effect of (8), the cardinality of the supporting set of optimal policies of sparse and soft MDP are compared at different values of $\alpha$.

To investigate effectiveness of the proposed method, we test sparsemax exploration and the sparse Bellman update rule on reinforcement learning with a continuous action space. To apply Q-learning to a continuous action space, a fine discretization is necessary to obtain a solution which is closer to the original continuous optimal policy. As the level of discretization increases, the number of actions to be explored becomes larger. In this regards, an efficient exploration method is required to obtain high performance. We compare our method to other exploration methods with respect to the convergence speed and the expected sum of rewards. We further check the effect of the update rule.

\section{A. Experiments on Performance Bounds and Supporting Set}

To verify our theorem about performance error bounds, we create a transition model $T$ by discretization of unicycle dynamics defined in a continuous state and action space and solve the original MDP, a soft MDP and a sparse MDP under predefined rewards while increasing the discretization level of the action space. The reward function is defined as a linear combination of two squared exponential functions, i.e., $\mathbf{r}(x)=\exp \left(\frac{\left\|x-x_{1}\right\|^{2}}{2 \sigma_{1}^{2}}\right)-\exp \left(\frac{\left\|x-x_{2}\right\|^{2}}{2 \sigma_{2}^{2}}\right)$, where $x$ is a location of a unicycle, $x_{1}$ is a goal point, $x_{2}$ is the point to avoid, and $\sigma_{1}$ and $\sigma_{2}$ are scale parameters. The reward function is designed to let an agent to navigate towards $x_{1}$ while avoiding $x_{2}$. The absolute value of differences between the expected return of the original MDP and that of sparse MDP (or soft MDP) is measured. As shown in Figure 2(a), the performance gap of sparse MDP converges to a constant bound while the performance of the soft MDP grows logarithmically. Note that the performance gaps of the sparse MDP and soft MDP are always smaller than their error bounds. Supporting set experiments are conducted using discretized unicycle dynamics. The cardinality of optimal policies are measured while $\alpha$ varies from 0.1 to 100 . In Figure 2(b), while the ratio of the supporting set for a soft MDP is changed from 0.79 to 1.00 , the ratio for a sparse MDP is changed from 0.24 to 0.99 , demonstrating the sparseness of the proposed sparse MDPs compared to soft MDPs.

\section{B. Reinforcement Learning in a Continuous Action Space}

We test our method in MuJoCo [22], a physics-based simulator, using two problems with a continuous action space: Inverted Pendulum and Reacher. The action space is discretized to apply Q-learning to a continuous action space and experiments are conducted with four different discretization levels to validate the effectiveness of sparsemax exploration and the sparse Bellman update rule. 
We compare the sparsemax exploration method to the $\epsilon$ greedy method and softmax exploration [10] and further compare the sparse Bellman update rule to the original Bellman update rule [20] and the soft Bellman update rule [11]. In addition, three different regularization coefficient settings are experimented. In total, we test 27 combinations of variants of deep Q-learning by combining three exploration methods, three update rules, and three different regularization coefficients of $0.01,0.1$, and 1 . The deep deterministic policy gradient (DDPG) method [15], which operates in a continuous action space without discretization of the action space, is also compare ${ }^{1}$. Hence, a total of 28 algorithms are tested.

Results are shown in Figure 3 and Figure 4 for inverted pendulum and reacher, respectively, where only the top five algorithms are plotted and each point in a graph is obtained by averaging the values from three independent runs with different random seeds. Results of all 28 algorithms are provided in Appendix B. Q network with two 512 dimensional hidden layers is used for the inverted pendulum problem and a network with four 256 dimensional hidden layers is used for the reacher problem. Each Q-learning algorithm utilizes the same network topology. For inverted pendulum, since the problem is easier than the reacher problem, most of top five algorithms converge to the maximum return of 1000 at each discretization level as shown in Figure 3(a). Four of top five algorithms utilize the proposed sparsemax exploration. Only one of the top five methods utilizes the softmax exploration. In Figure 3(b) the number of episodes required to reach a near optimal return, 980 , is shown. The sparsemax exploration requires a less number of episodes to obtain a near optimal value than $\epsilon$-greedy, softmax exploration.

For the reacher problem, the algorithms with sparsemax exploration slightly outperforms $\epsilon$-greedy methods and the performance of softmax exploration is not included in the top five as shown in Figure 4(a). In terms of the number of required episodes, sparsemax exploration outperforms epsilon greedy methods as shown in Figure 4(b), where we set the threshold return to be -6 . DDPG shows poor performances in both problems since the number of sampled episodes is insufficient. In this regards, deep Q-learning with sparsemax exploration outperforms DDPG with less number of episodes. From these experiments, it can be known that the sparsemax exploration method has an advantage over softmax exploration, $\epsilon$-greedy method and DDPG with respect to the number of episodes required to reach the optimal performance.

\section{CONCLUSION}

In this paper, we have proposed a new MDP with novel causal sparse Tsallis entropy regularization which induces a sparse and multi-modal optimal policy distribution. In addition, we have provided the full mathematical analysis of the proposed sparse MDPs: the optimality condition of sparse MDPs given as the sparse Bellman equation, sparse value iteration and its convergence and optimality properties, and the performance bounds between the propose MDP and the

${ }^{1}$ To test DDPG, we used the code from Open AI available at https://github. com/openai/baselines

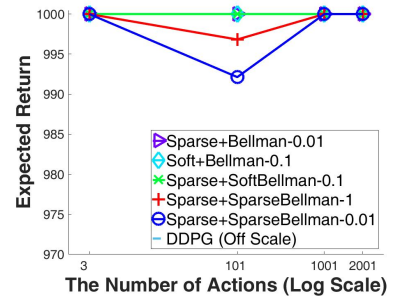

(a) Expected Return

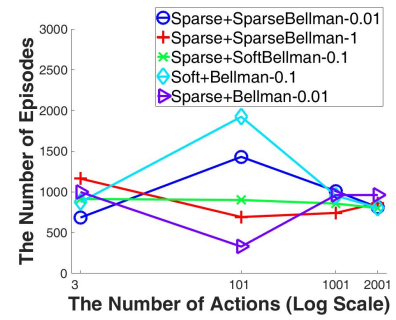

(b) Required Episodes
Fig. 3: Inverted pendulum problem. Algorithms are named as <exploration method $\rangle+\langle$ update rule $\rangle+\langle\alpha\rangle$. (a) The average performance of each algorithm after 3000 episodes. The performance of DDPG is out of scale. (b) The average number of episodes required to reach the threshold value 980 .

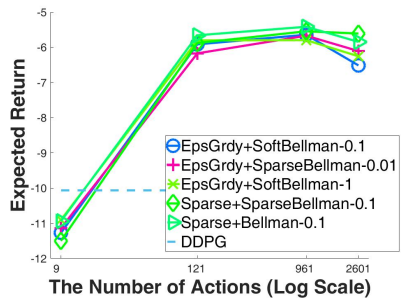

(a) Expected Return

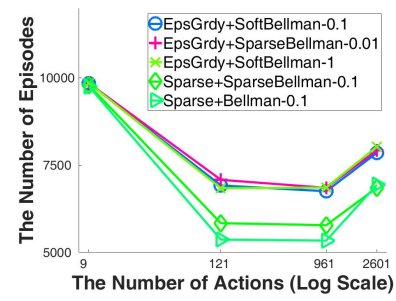

(b) Required Episodes
Fig. 4: Reacher problem. (a) The average performance of each algorithm after 10000 episodes. (b) The average number of episodes required to reach the threshold value -6 .

original MDP. We have also proven that the performance gap of a sparse MDP is strictly smaller than that of a soft MDP. In experiments, we have verified that the theoretical performance gaps of a sparse MDP and soft MDP from the original MDP are correct. We have applied the sparsemax policy and sparse Bellman equation to deep Q-learning as the exploration strategy and update rule, respectively, and shown that the proposed exploration method shows significantly better performance compared to $\epsilon$-greedy, softmax exploration, and DDPG, as the number of actions increases. From the analysis and experiments, we have demonstrated that the proposed sparse MDP can be an efficient alternative to problems with a large number of possible actions and even a continuous action space.

\section{APPENDIX A}

\section{A. Sparse Bellman Equation from Karush-Kuhn-Tucker con-} ditions

The following proof explains the optimality condition of the sparse MDP from Karush-Kuhn-Tucker (KKT) conditions.

Proof of Theorem 17. The KKT conditions of (3) are as follows:

$$
\begin{array}{ll}
\forall s, a & \sum_{a^{\prime}} \pi\left(a^{\prime} \mid s\right)-1=0, \quad-\pi(a \mid s) \leq 0 \\
\forall s, a & \lambda_{s a} \geq 0 \\
\forall s, a & \lambda_{s a} \pi(a \mid s)=0 \\
\forall s, a & \frac{\partial L(\pi, c, \lambda)}{\partial \pi(a \mid s)}=0
\end{array}
$$


where $c$ and $\lambda$ are Lagrangian multipliers for the equality and inequality constraints, respectively, and $(9)$ is the feasibility of primal variables, (10) is the feasibility of dual variables, (11) is the complementary slackness and (12) is the stationarity condition. The Lagrangian function of (3) is written as follows:

$$
\begin{aligned}
& L(\pi, c, \lambda) \\
& =-J_{\pi}^{s p}+\sum_{s} c_{s}\left(\sum_{a^{\prime}} \pi\left(a^{\prime} \mid s\right)-1\right)-\sum_{s, a} \lambda_{s a} \pi(a \mid s)
\end{aligned}
$$

where the maximization of (3) is changed into the minimization problem, i.e., $\min _{\pi}-J_{\pi}^{s p}$. First, the derivative of $J_{\pi}^{s p}$ can be obtained by using the chain rule.

$$
\begin{aligned}
& \frac{\partial J_{\pi}}{\partial \pi(a \mid s)}=d^{\top} G_{\pi}^{-1} \frac{\partial r_{\pi}^{s p}}{\partial \pi(a \mid s)}+\gamma d^{\top} G_{\pi}^{-1} \frac{\partial T_{\pi}}{\partial \pi(a \mid s)} G_{\pi}^{-1} r_{\pi}^{s p} \\
& =\rho_{\pi}^{\top} \frac{\partial r_{\pi}^{s p}}{\partial \pi(a \mid s)}+\gamma \rho_{\pi}^{\top} \frac{\partial T_{\pi}}{\partial \pi(a \mid s)} V_{\pi}^{s p} \\
& =\rho_{\pi}(s)\left(r(s, a)+\frac{\alpha}{2}-\alpha \pi(a \mid s)+\gamma \sum_{s^{\prime}} V_{\pi}^{s p}\left(s^{\prime}\right) T\left(s^{\prime} \mid s, a\right)\right) \\
& =\rho_{\pi}(s)\left(Q_{\pi}^{s p}(s, a)+\frac{\alpha}{2}-\alpha \pi(a \mid s)\right) .
\end{aligned}
$$

Here, the partial derivative of Lagrangian is obtained as follows:

$$
\begin{aligned}
& \frac{\partial L(\pi, c, \lambda)}{\partial \pi(a \mid s)} \\
& =-\rho_{\pi}(s)\left(Q_{\pi}^{s p}(s, a)+\frac{\alpha}{2}-\alpha \pi(a \mid s)\right)+c_{s}-\lambda_{s a}=0 .
\end{aligned}
$$

First, consider a positive $\pi(a \mid s)$ where the corresponding Lagrangian multiplier $\lambda_{s a}$ is zero due to the complementary slackness. By summing $\pi(a \mid s)$ with respect to action $a$, Lagrangian multiplier $c_{s}$ can be obtained as follows:

$$
\begin{aligned}
& 0=-\rho_{\pi}(s)\left(Q_{\pi}^{s p}(s, a)+\frac{\alpha}{2}-\alpha \pi(a \mid s)\right)+c_{s} \\
& \pi(a \mid s)=\left(-\frac{c_{s}}{\rho_{\pi}(s) \alpha}+\frac{1}{2}+\frac{Q_{\pi}^{s p}(s, a)}{\alpha}\right) \\
& \sum_{\pi\left(a^{\prime} \mid s\right)>0} \pi\left(a^{\prime} \mid s\right)=\sum_{\pi\left(a^{\prime} \mid s\right)>0}\left(-\frac{c_{s}}{\rho_{\pi}(s) \alpha}+\frac{1}{2}+\frac{Q_{\pi}^{s p}\left(s, a^{\prime}\right)}{\alpha}\right)=1 \\
& \therefore c_{s}=\rho_{\pi}(s) \alpha\left[\frac{\sum_{\pi\left(a^{\prime} \mid s\right)>0} \frac{Q_{\pi}^{s p}\left(s, a^{\prime}\right)}{\alpha}-1}{K}+\frac{1}{2}\right]
\end{aligned}
$$

where $K$ is the number of positive elements of $\pi(\cdot \mid s)$. By replacing $c_{s}$ with this result, the optimal policy distribution is induced as follows.

$$
\begin{aligned}
\pi(a \mid s) & =\left(-\frac{c_{s}}{\rho_{\pi}(s) \alpha}+\frac{1}{2}+\frac{Q_{\pi}^{s p}(s, a)}{\alpha}\right) \\
& =\frac{Q_{\pi}^{s p}(s, a)}{\alpha}-\frac{\sum_{\pi\left(a^{\prime} \mid s\right)>0} \frac{Q_{\pi}^{s p}\left(s, a^{\prime}\right)}{\alpha}-1}{K}
\end{aligned}
$$

As this equation is derived under the assumption that $\pi(a \mid s)$ is positive. For $\pi(a \mid s)>0$, following condition is necessarily fulfilled,

$$
\frac{Q_{\pi}^{s p}(s, a)}{\alpha}>\frac{\sum_{\pi\left(a^{\prime} \mid s\right)>0} \frac{Q_{\pi}^{s p}\left(s, a^{\prime}\right)}{\alpha}-1}{K} .
$$

We notate this supporting set as $S(s)=\left\{a \mid 1+K \frac{Q_{\pi}^{s p}(s, a)}{\alpha}>\right.$ $\left.\sum_{\pi\left(a^{\prime} \mid s\right)>0} \frac{Q_{\pi}^{s p}\left(s, a^{\prime}\right)}{\alpha}\right\} . S(s)$ contains the actions which has larger action values than threshold

$$
\tau\left(Q_{\pi}^{s p}(s, \cdot)\right)=\frac{\sum_{\pi\left(a^{\prime} \mid s\right)>0} \frac{Q_{\pi}^{s p}\left(s, a^{\prime}\right)}{\alpha}-1}{K} .
$$

By using these notations, the optimal policy distribution can be rewritten as follows:

$$
\pi(a \mid s)=\max \left(\frac{Q_{\pi}^{s p}(s, a)}{\alpha}-\tau\left(\frac{Q_{\pi}^{s p}(s, \cdot)}{\alpha}\right), 0\right) .
$$

By substituting $\pi(a \mid s)$ with this result, the following optimality equation of $V_{\pi}^{s p}$ is induced.

$$
\begin{aligned}
& V_{\pi}^{s p}(s) \\
& =\sum_{a} \pi(a \mid s)\left(Q_{\pi}^{s p}(s, a)+\frac{\alpha}{2}(1-\pi(a \mid s))\right) \\
& =\sum_{a} \pi(a \mid s)\left(Q_{\pi}^{s p}(s, a)-\frac{\alpha}{2} \pi(a \mid s)\right)+\frac{\alpha}{2} \sum_{a} \pi(a \mid s) \\
& =\sum_{a \in S(s)} \pi(a \mid s) \\
& \times\left(Q_{\pi}^{s p}(s, a)-\frac{\alpha}{2}\left(\frac{Q_{\pi}^{s p}(s, a)}{\alpha}-\tau\left(\frac{Q_{\pi}^{s p}(s, \cdot)}{\alpha}\right)\right)\right)+\frac{\alpha}{2} \\
& =\sum_{a \in S(s)} \pi(a \mid s) \frac{\alpha}{2}\left(\frac{Q_{\pi}^{s p}(s, a)}{\alpha}+\tau\left(\frac{Q_{\pi}^{s p}(s, \cdot)}{\alpha}\right)\right)+\frac{\alpha}{2} \\
& =\alpha\left[\frac{1}{2} \sum_{a \in S(s)}^{K}\left(\left(\frac{Q_{\pi}^{s p}(s, a)}{\alpha}\right)^{2}-\tau\left(\frac{Q_{\pi}^{s p}(s, \cdot)}{\alpha}\right)^{2}\right)+\frac{1}{2}\right]
\end{aligned}
$$

To summarize, we obtain the sparse Bellman equation as follows:

$$
\begin{aligned}
Q_{\pi}^{s p}(s, a) & =r(s, a)+\gamma \sum_{s^{\prime}} V_{\pi}^{s p}\left(s^{\prime}\right) T\left(s^{\prime} \mid s, a\right) \\
V_{\pi}^{s p}(s) & =\alpha\left[\frac{1}{2} \sum_{a \in S(s)}^{K}\left(\left(\frac{Q_{\pi}^{s p}(s, a)}{\alpha}\right)^{2}-\tau\left(\frac{Q_{\pi}^{s p}(s, \cdot)}{\alpha}\right)^{2}\right)+\frac{1}{2}\right] \\
\pi(a \mid s) & =\max \left(\frac{Q_{\pi}^{s p}(s, a)}{\alpha}-\tau\left(\frac{Q_{\pi}^{s p}(s, \cdot)}{\alpha}\right), 0\right) .
\end{aligned}
$$

\section{B. Upper and Lower Bounds for Sparsemax Operation}

In this section, we prove the lower and upper bounds of $\operatorname{spmax}(z)$ defined in $(6)$. We would like to mention that the proof of lower bound of (7) is provided in [17]. However, we find another interesting way to prove (7) by using the Cauchy-Schwartz inequality and the nonnegative property of a quadratic equation.

We first prove $\max (z) \leq \operatorname{spmax}(z)$ and next prove $\operatorname{spmax}(z) \leq \max (z)+\frac{d-1}{2 d}$. Without loss of generality, we assume that $\alpha=1$ but the original inequalities can be simply obtained by replacing $z$ with $\frac{z}{\alpha}$.

Lower Bound of SparseMax Operation. For all $z \in \mathbb{R}^{d}$, $\max (z) \leq \operatorname{spmax}(z)$ holds.

Proof: We prove that, for all $z, \operatorname{spmax}(z)-z_{(1)} \geq 0$ where $z_{(1)}=\max (z)$ by definition. The proof is done by 
simply rearranging the terms in $(6)$,

$$
\begin{aligned}
& \operatorname{spmax}(z)-z_{(1)} \\
& =\frac{1}{2} \sum_{i=1}^{K}\left(z_{(i)}^{2}-\tau(z)^{2}\right)+\frac{1}{2}-z_{(1)} \\
& =\frac{1}{2} \sum_{i=1}^{K} z_{(i)}^{2}-\frac{K}{2}\left(\frac{\sum_{i=1}^{K} z_{(i)}-1}{K}\right)^{2}+\frac{1}{2}-z_{(1)} \\
& =\frac{1}{2} \sum_{i=1}^{K} z_{(i)}^{2}-\frac{1}{2 K}\left(\sum_{i=1}^{K} z_{(i)}-1\right)^{2}+\frac{1}{2}-z_{(1)} \\
& =\frac{K \sum_{i=1}^{K} z_{(i)}^{2}-\left(\sum_{i=1}^{K} z_{(i)}-1\right)^{2}-2 K z_{(1)}+K}{2 K} \\
& =\frac{1}{2 K}\left(K z_{(1)}^{2}+K \sum_{i=2}^{K} z_{(i)}^{2}\right. \\
& \left.-\left(z_{(1)}+\sum_{i=2}^{K} z_{(i)}-1\right)^{2}-2 K z_{(1)}+K\right) .
\end{aligned}
$$

The quadratic term can be decomposed as follows:

$$
\begin{aligned}
& \left(z_{(1)}+\sum_{i=2}^{K} z_{(i)}-1\right)^{2} \\
& =z_{(1)}^{2}+\left(\sum_{i=2}^{K} z_{(i)}\right)^{2}+1+2 z_{(1)} \sum_{i=2}^{K} z_{(i)}-2 z_{(1)}-2 \sum_{i=2}^{K} z_{(i)} .
\end{aligned}
$$

By putting this result into the equation and rearranging them, three terms are obtained as follows:

$$
\begin{aligned}
& \operatorname{spmax}(z)-z_{(1)} \\
& =\frac{1}{2 K}\left((K-1) z_{(1)}^{2}-2 z_{(1)}\left\{\sum_{i=2}^{K} z_{(i)}+K-1\right\}\right. \\
& \left.+K \sum_{i=2}^{K} z_{(i)}^{2}+2 \sum_{i=2}^{K} z_{(i)}+K-\left(\sum_{i=2}^{K} z_{(i)}\right)^{2}\right) .
\end{aligned}
$$

Then, $K \sum_{i=2}^{K} z_{(i)}^{2}+2 \sum_{i=2}^{K} z_{(i)}+K$ can be replaced with $K \sum_{i=2}^{K}\left(z_{(i)}+1\right)^{2}-2(K-1) \sum_{i=2}^{K} z_{(i)}$ and we also decompose the second term $-2 z_{(1)}\left\{\sum_{i=2}^{K} z_{(i)}+K-1\right\}$ into two parts: $-2 z_{(1)}\left\{\sum_{i=2}^{K}\left(z_{(i)}+1\right)\right\}$ and $2 z_{(1)}$, and rearrange the equation as follows,

$$
\begin{aligned}
& =\frac{1}{2 K}\left((K-1) z_{(1)}^{2}-2 z_{(1)}\left\{\sum_{i=2}^{K}\left(z_{(i)}+1\right)\right\}\right. \\
& \left.+K \sum_{i=2}^{K}\left(z_{(i)}+1\right)^{2}-2(K-1) \sum_{i=2}^{K} z_{(i)}-\left(\sum_{i=2}^{K} z_{(i)}\right)^{2}\right) .
\end{aligned}
$$

Again, we change $-2(K-1) \sum_{i=2}^{K} z_{(i)}-\left(\sum_{i=2}^{K} z_{(i)}\right)^{2}$ into $-\left(\sum_{i=2}^{K}\left(z_{(i)}+1\right)\right)^{2}+(K-1)^{2}$ by adding and subtracting $(K-1)^{2}$ as follow,

$$
\begin{aligned}
& =\frac{1}{2 K}\left((K-1) z_{(1)}^{2}-2 z_{(1)}\left\{\sum_{i=2}^{K}\left(z_{(i)}+1\right)\right\}\right. \\
& \left.+K \sum_{i=2}^{K}\left(z_{(i)}+1\right)^{2}-\left(\sum_{i=2}^{K}\left(z_{(i)}+1\right)\right)^{2}+(K-1)^{2}\right) .
\end{aligned}
$$

Then, the term $(K-1) z_{(1)}^{2}-2 z_{(1)}\left\{\sum_{i=2}^{K}\left(z_{(i)}+1\right)\right\}$ is reformulated as $(K-1)\left(z_{(1)}-\frac{\sum_{i=2}^{K}\left(z_{(i)}+1\right)}{K-1}\right)^{2}-(K-$ 1) $\left(\frac{\sum_{i=2}^{K}\left(z_{(i)+1}\right)}{K-1}\right)^{2}$. By using this reformulation, we can obtain following equation.

$$
\begin{aligned}
& =\frac{(K-1)}{2 K}\left[z_{(1)}-\frac{\sum_{i=2}^{K}\left(z_{(i)}+1\right)}{K-1}\right]^{2}+ \\
& \frac{1}{2 K}\left(-\frac{\left(\sum_{i=2}^{K}\left(z_{(i)+1}\right)\right)^{2}}{K-1}+K \sum_{i=2}^{K}\left(z_{(i)}+1\right)^{2}-\left(\sum_{i=2}^{K}\left(z_{(i)}+1\right)\right)^{2}\right. \\
& \left.+(K-1)^{2}\right)
\end{aligned}
$$

Finally, we can obtain three terms by rearranging the above equation,

$$
\begin{aligned}
& =\frac{(K-1)}{2 K}\left[z_{(1)}-\frac{\sum_{i=2}^{K}\left(z_{(i)}+1\right)}{K-1}\right]^{2} \\
& +\frac{1}{2 K}\left(K \sum_{i=2}^{K}\left(z_{(i)}+1\right)^{2}-K \frac{\left(\sum_{i=2}^{K}\left(z_{(i)}+1\right)\right)^{2}}{K-1}\right)+\frac{(K-1)^{2}}{2 K} \\
& =\frac{(K-1)}{2 K}\left[z_{(1)}-\frac{\sum_{i=2}^{K}\left(z_{(i)}+1\right)}{K-1}\right]^{2} \\
& +\frac{K-1}{2}\left[\sum_{i=2}^{K} \frac{\left(z_{(i)}+1\right)^{2}}{K-1}-\left(\sum_{i=2}^{K} \frac{\left(z_{(i)}+1\right)}{K-1}\right)^{2}\right]+\frac{(K-1)^{2}}{2 K}
\end{aligned}
$$

where the first and third terms are quadratic and always nonnegative. The second term is also always nonnegative by the Cauchy-Schwartz inequality. The Cauchy-Schwartz inequality is written as $\left(\mathbf{p}^{\top} \mathbf{q}\right)^{2} \leq\|\mathbf{p}\|^{2}\|\mathbf{q}\|^{2}$. Let $z_{2: K}=$ $\left[z_{(2)}, \cdots, z_{(K)}\right]^{\top}$, then, by setting $\mathbf{p}=z_{2: K}+\mathbf{1}$ and $\mathbf{q}=$ $\frac{1}{K-1} \mathbf{1}$ where 1 is a $K-1$ dimensional vector of ones, it can be shown that the second term is nonnegative. Therefore, $\operatorname{spmax}(z)-z_{(1)}$ is always nonnegative for all $z$ since three remaining terms are always nonnegative, completing the proof.

Now, we prove the upper bound of sparsemax operation.

Upper Bound of SparseMax Operation. For all $z \in \mathbb{R}^{d}$, $\operatorname{spmax}(z) \leq \max (z)+\frac{d-1}{2 d}$ holds.

Proof: First, we decompose the summation of (6) into 
two terms as follows:

$$
\begin{aligned}
& \operatorname{spmax}(z)=\frac{1}{2} \sum_{i=1}^{K}\left(z_{(i)}^{2}-\tau(z)^{2}\right)+\frac{1}{2} \\
& =\frac{1}{2} \sum_{i=1}^{K}\left(z_{(i)}-\tau(z)\right)\left(z_{(i)}+\tau(z)\right)+\frac{1}{2} \\
& \leq \frac{1}{2} \sum_{i=1}^{K} p_{i}^{*}(z)\left(z_{(i)}+\tau(z)\right)+\frac{1}{2} \\
& =\frac{1}{2} \sum_{i=1}^{K} p_{i}^{*}(z) z_{(i)}+\frac{\tau(z)}{2} \sum_{i=1}^{K} p_{i}^{*}(z)+\frac{1}{2} \\
& =\frac{1}{2} \sum_{i=1}^{K} p_{i}^{*}(z) z_{(i)}+\frac{\tau(z)}{2}+\frac{1}{2} \\
& =\frac{1}{2} \sum_{i=1}^{K} p_{i}^{*}(z) z_{(i)}+\frac{1}{2} \sum_{i=1}^{K} \frac{z_{(i)}}{K}-\frac{1}{2 K}+\frac{1}{2}
\end{aligned}
$$

where $p_{i}^{*}=\max \left(z_{(i)}-\tau(z), 0\right)$ which is the optimal solution of the simplex projection problem $\sqrt{5}$ and $\sum_{i=1}^{K} p_{i}^{*}(z)=1$ by definition. Now, we use the fact that, for every $p$ on $d-1$ dimensional simplex, $\sum_{i}^{d} p_{i} z_{i} \leq \max (z)$ for all $z \in \mathbb{R}^{d}$. By using this property, as $p^{*}(z)$ and $\frac{1}{K} \mathbf{1}$ are on the probability simplex, following inequality is induced,

$$
\begin{aligned}
& \operatorname{spmax}(z)=\frac{1}{2} \sum_{i=1}^{K} p_{i}^{*}(z) z_{(i)}+\frac{1}{2} \sum_{i=1}^{K} \frac{z_{(i)}}{K}-\frac{1}{2 K}+\frac{1}{2} \\
& \leq \frac{1}{2} \max (z)+\frac{1}{2} \max (z)-\frac{1}{2 K}+\frac{1}{2} \leq \max (z)-\frac{1}{2 K}+\frac{1}{2} \\
& \leq \max (z)-\frac{1}{2 d}+\frac{1}{2}
\end{aligned}
$$

where $d \geq K$ by definition of $K$. Therefore, $\operatorname{spmax}(z) \leq$ $\max (z)+\frac{d-1}{2 d}$ holds.

\section{Comparison to Log-Sum-Exp}

We explain the error bounds for the log-sum-exp operation and compare it to the bounds of the sparsemax operation. The log-sum-exp operation has widely known bounds,

$$
\max (z) \leq \operatorname{logsumexp}(z) \leq \max (z)+\log (d) .
$$

We would like to note that sparsemax has tighter bounds than log-sum-exp as it is always satisfied that, for all $d>1$, $\frac{d-1}{2 d} \leq \log (d)$. Intuitively, the approximation error of $\log$ sum-exp increases as the dimension of input space increases. However, the approximation error of sparsemax approaches to $\frac{1}{2}$ as the dimension of input space goes infinity. This fact plays a crucial role in comparing performance error bounds of the sparse MDP and soft MDP.

\section{Causal Sparse Tsallis Entropy}

The following proof shows that $W(\pi)$ is equivalent to the discounted expected sum of special case of Tsallis entropy when $q=2$ and $k=\frac{1}{2}$.
Proof of Theorem 2. The proof is simply done by rewriting our regularization as follows:

$$
\begin{aligned}
& W(\pi) \\
& =\mathbb{E}\left[\sum_{t=0}^{\infty} \gamma^{t} \frac{1}{2}\left(1-\pi\left(a_{t} \mid s_{t}\right)\right) \mid \pi, d, T\right] \\
& =\sum_{s, a} \frac{1}{2}(1-\pi(a \mid s)) \mathbb{E}\left[\sum_{t=0}^{\infty} \gamma^{t} \mathbb{1}_{\left\{s_{t}=s, a_{t}=a\right\}} \mid \pi, d, T\right] \\
& =\sum_{s, a} \frac{1}{2}(1-\pi(a \mid s)) \rho_{\pi}(s, a) \\
& =\sum_{s} \rho_{\pi}(s) \sum_{a} \frac{1}{2}(1-\pi(a \mid s)) \pi(a \mid s) \\
& =\sum_{s} \rho_{\pi}(s) \frac{1}{2}\left(\sum_{a} \pi(a \mid s)-\sum_{a} \pi(a \mid s)^{2}\right) \\
& =\sum_{s} \rho_{\pi}(s) \frac{1}{2}\left(1-\sum_{a} \pi(a \mid s)^{2}\right) \\
& =\sum_{s} S_{2, \frac{1}{2}}(\pi(\cdot \mid s)) \rho_{\pi}(s)=\mathbb{E}_{\pi}\left[S_{2, \frac{1}{2}}(\pi(\cdot \mid s))\right]
\end{aligned}
$$

\section{E. Convergence and Optimality of Sparse Value Iteration}

In this section, the monotonicity, discounting property, contraction of sparse Bellman operation $U^{s p}$ are proved.

Proof of Lemma 17. In [17], the monotonicity of (6) is proved. Then, the monotonicity of $U^{s p}$ can be proved using (6). Let $x$ and $y$ are given such that $x \leq y$. Then,

$\frac{r(s, a)+\gamma \sum_{s^{\prime}} x\left(s^{\prime}\right) T\left(s^{\prime} \mid s, a\right)}{\alpha} \leq \frac{r(s, a)+\gamma \sum_{s^{\prime}} y\left(s^{\prime}\right) T\left(s^{\prime} \mid s, a\right)}{\alpha}$

where $T\left(s^{\prime} \mid s, a\right)$ is a transition probability which is always nonnegative. Since the sparsemax operation is monotone, the following inequality is induced

$$
\begin{aligned}
& \alpha \operatorname{spmax}\left(\frac{r(s, a)+\gamma \sum_{s^{\prime}} x\left(s^{\prime}\right) T\left(s^{\prime} \mid s, a\right)}{\alpha}\right) \\
& \leq \alpha \operatorname{spmax}\left(\frac{r(s, a)+\gamma \sum_{s^{\prime}} y\left(s^{\prime}\right) T\left(s^{\prime} \mid s, a\right)}{\alpha}\right) .
\end{aligned}
$$

Finally, we can obtain

$$
\therefore U^{s p}(x) \leq U^{s p}(y) .
$$

Proof of Lemma 2, In [17], it is shown that for $c \in \mathbb{R}$ and $x \in \mathbb{R}^{|\mathcal{S}|}, \operatorname{spmax}(x+c \mathbf{1})=\operatorname{spmax}(x)+c \mathbf{1}$. Using this property,

$$
\begin{aligned}
& U^{s p}(x+c \mathbf{1})(s) \\
& =\alpha \operatorname{spmax}\left(\frac{r(s, a)+\gamma \sum_{s^{\prime}}\left(x\left(s^{\prime}\right)+c\right) T\left(s^{\prime} \mid s, a\right)}{\alpha}\right) \\
& =\alpha \operatorname{spmax}\left(\frac{r(s, a)+\gamma \sum_{s^{\prime}} x\left(s^{\prime}\right) T\left(s^{\prime} \mid s, a\right)+\gamma c \sum_{s^{\prime}} T\left(s^{\prime} \mid s, a\right)}{\alpha}\right) \\
& =\alpha \operatorname{spmax}\left(\frac{r(s, a)+\gamma \sum_{s^{\prime}} x\left(s^{\prime}\right) T\left(s^{\prime} \mid s, a\right)}{\alpha}+\frac{\gamma c}{\alpha}\right) \\
& =\alpha \operatorname{spmax}\left(\frac{r(s, a)+\gamma \sum_{s^{\prime}} x\left(s^{\prime}\right) T\left(s^{\prime} \mid s, a\right)}{\alpha}\right)+\gamma c \\
& \therefore U^{s p}(x+c \mathbf{1})=U^{s p}(x)+\gamma c \mathbf{1} .
\end{aligned}
$$


Proof of Lemma 3. First, we prove that $U^{s p}$ is a $\gamma$ contraction mapping with respect to $d_{\max }$. Without loss of generality, the proof is discussed for a general function $\phi$ : $\mathbb{R}^{|\mathcal{S}|} \rightarrow \mathbb{R}^{|\mathcal{S}|}$ with discounting and monotone properties.

Let $d_{\max }(x, y)=M$. Then, $y-M \mathbf{1} \leq x \leq y+M \mathbf{1}$ is satisfied. By monotone and discounting properties, the following inequality between mappings $\phi(x)$ and $\phi(y)$ is established.

$$
\phi(y)-\gamma M \mathbf{1} \leq \phi(x) \leq \phi(y)+\gamma M \mathbf{1},
$$

where $\gamma$ is a discounting factor of $\phi$. From this inequality, $d_{\max }(\phi(x), \phi(y)) \leq \gamma M=\gamma d_{\max }(x, y)$ and $\gamma \in(0,1)$. Therefore, $\phi$ is a $\gamma$-contraction mapping. In our case, $U^{s p}$ is a $\gamma$-contraction mapping.

As $\mathbb{R}^{|\mathcal{S}|}$ and $d_{\max }(x, y)$ are a non-empty complete metric space, by Banach fixed-point theorem, a $\gamma$-contraction mapping $U^{s p}$ has a unique fixed point.

Using Lemma 1, Lemma 2, and Lemma 3, we can prove the convergence and optimality of sparse value iteration.

Proof of Theorem 3. Sparse value iteration converges into a fixed point of $U^{s p}$ by the contraction property. Let $x_{*}$ be a fixed point of $U^{s p}$ and, by definition of $U^{s p}, x_{*}$ is the point that satisfies the sparse Bellman equation, i.e. $x_{*}=U^{s p}\left(x_{*}\right)$. Hence, by Theorem 1. $x_{*}$ satisfies necessity conditions of the optimal solution. By the Banach fixed point theorem, $x_{*}$ is a unique point which satisfies necessity conditions of optimal solution. In particular, $x_{*}=U^{s p}\left(x_{*}\right)$ is precisely equivalent to the sparse Bellman equation. In other words, there is no other point that satisfies the sparse Bellman equation. Therefore, $x_{*}$ is the optimal value of a sparse MDP.

\section{F. Performance Error Bounds for Sparse Value Iteration}

In this section, we prove the performance error bounds for sparse value iteration and soft value iteration. We first show that the optimal values of a sparse MDP and a soft MDP are greater than that of the original MDP.

Proof of Lemma 4. We first prove the inequality of the sparse Bellman operation

$$
U^{n}(x) \leq\left(U^{s p}\right)^{n}(x), \quad x_{*} \leq x_{*}^{s p} .
$$

This inequality can be proven by the mathematical induction. When $n=1$, the inequality is proven as follows:

$$
\begin{gathered}
\max _{a^{\prime}}\left(r\left(s, a^{\prime}\right)+\gamma \sum_{s^{\prime}} x\left(s^{\prime}\right) T\left(s^{\prime} \mid s, a^{\prime}\right)\right) \\
\leq \operatorname{spmax}\left(r(s, \cdot)+\gamma \sum_{s^{\prime}} x\left(s^{\prime}\right) T\left(s^{\prime} \mid s, \cdot\right)\right) \\
(\because \max (z) \leq \operatorname{spmax}(z)) .
\end{gathered}
$$

Therefore,

$$
U(x) \leq U^{s p}(x) .
$$

For some positive integer $k$, let us assume that $U^{k}(x) \leq$ $\left(U^{s p}\right)^{k}(x)$ holds for every $x \in \mathbb{R}^{|\mathcal{S}|}$. Then, when $n=k+1$,

$$
\begin{aligned}
U^{k+1}(x) & =U^{k}(U(x)) \\
& \leq\left(U^{s p}\right)^{k}(U(x)) \quad\left(\because U^{k}(x) \leq\left(U^{s p}\right)^{k}(x)\right) \\
& \leq\left(U^{s p}\right)^{k}\left(U^{s p}(x)\right) \quad\left(\because U(x) \leq U^{s p}(x)\right) \\
& =\left(U^{s p}\right)^{k+1}(x) .
\end{aligned}
$$

Therefore, by mathematical induction, it is satisfied $U^{n}(x) \leq$ $\left(U^{s p}\right)^{n}(x)$ for every positive integer $n$. Then, the inequality of the fixed points of $U$ and $U^{s p}$ can be obtained by $n \rightarrow \infty$,

$$
x_{*} \leq x_{*}^{s p}
$$

where $*$ indicates the fixed point. The above arguments also hold when $U^{s p}$ and sparsemax are replaced with $U^{\text {soft }}$ and log-sum-exp operation, respectively.

Before showing the performance error bounds, the upper bounds of $W(\pi)$ and $H(\pi)$ are proved first.

Proof of Lemma 5 . For $W(\pi)$,

$$
\begin{aligned}
& W(\pi)=\sum_{s} \rho_{\pi}(s) \sum_{a} \frac{1}{2}(1-\pi(a \mid s)) \pi(a \mid s) \\
& \leq \sum_{s} \rho_{\pi}(s) \frac{|\mathcal{A}|-1}{2|\mathcal{A}|}\left(\because \sum_{a} \frac{1}{2}(1-\pi(a \mid s)) \pi(a \mid s) \leq \frac{|\mathcal{A}|-1}{2|\mathcal{A}|}\right) \\
& =\frac{1}{1-\gamma} \frac{|\mathcal{A}|-1}{2|\mathcal{A}|}\left(\because \sum_{s} \rho_{\pi}(s)=\frac{1}{1-\gamma}\right) .
\end{aligned}
$$

The inequality that $\sum_{a} \frac{1}{2}(1-\pi(a \mid s)) \pi(a \mid s) \leq \frac{|\mathcal{A}|-1}{2|\mathcal{A}|}$ can be obtained by finding the point where the derivative of $\frac{1}{2}(1-x) x$ is zero. Similarly, for $H(\pi)$,

$$
\begin{aligned}
& H(\pi)=\mathbb{E}\left[\sum_{t=0}^{\infty} \gamma^{t}-\log \left(\pi\left(a_{t} \mid s_{t}\right)\right) \mid \pi, d, T\right] \\
& =\sum_{s, a}-\log (\pi(a \mid s)) \mathbb{E}\left[\sum_{t=0}^{\infty} \gamma^{t} \mathbb{1}_{\left\{s_{t}=s, a_{t}=a\right\}} \mid \pi, d, T\right] \\
& =\sum_{s, a}-\log (\pi(a \mid s)) \rho_{\pi}(s, a) \\
& =\sum_{s} \rho_{\pi}(s) \sum_{a}-\log (\pi(a \mid s)) \pi(a \mid s) \\
& \leq \sum_{s} \rho_{\pi}(s) \log (|\mathcal{A}|)\left(\because \sum_{a}-\log (\pi(a \mid s)) \pi(a \mid s) \leq \log (|\mathcal{A}|)\right) \\
& =\frac{1}{1-\gamma} \log (|\mathcal{A}|) \quad\left(\because \sum_{s} \rho_{\pi}(s)=\frac{1}{1-\gamma}\right) .
\end{aligned}
$$

The inequality that $\sum_{a}-\log (\pi(a \mid s)) \pi(a \mid s) \leq \log (|\mathcal{A}|)$ also can be obtained by finding the point where the derivative of $-x \log (x)$ is zero.

Using Lemma 4 and Lemma 5 , the error bounds of sparse and soft value iterations can be proved.

Proof of Theorem 4. Let $\pi_{*}$ be the optimal policy of the original MDP, where the problem is defined as $\max _{\pi} \mathbb{E}_{\pi}(\mathbf{r}(s, a))$.

$$
\mathbb{E}_{\pi_{*}^{s p}}(\mathbf{r}(s, a)) \leq \max _{\pi} \mathbb{E}_{\pi}(\mathbf{r}(s, a))=\mathbb{E}_{\pi_{*}}(\mathbf{r}(s, a)) .
$$

The rightside inequality is by the definition of optimality. Before proving the leftside inequality, we first derive the following inequality from Lemma 4.

$$
V_{*} \leq V_{*}^{s p},
$$

where $*$ indicates an optimal value. Since the fixed points of $U$ and $U^{s p}$ are the optimal solutions of the original MDP and sparse MDP, respectively, 13) can be derived from Lemma 4 
The leftside inequality is proved using (13) as follows:

$$
\begin{aligned}
& \mathbb{E}_{\pi_{*}}(\mathbf{r}(s, a))=d^{\top} V_{*} \\
& \leq d^{\top} V_{*}^{s p}=J_{*}^{s p}=\mathbb{E}_{\pi_{*}^{s p}}(\mathbf{r}(s, a))+\alpha W\left(\pi_{*}^{s p}\right) \\
& \leq \mathbb{E}_{\pi_{*}^{s p}}(\mathbf{r}(s, a))+\frac{\alpha}{1-\gamma} \frac{|\mathcal{A}|-1}{2|\mathcal{A}|}(\because \text { Lemma } 5) .
\end{aligned}
$$

Proof of Theorem 5. Let $\pi_{*}$ be the optimal policy of the original MDP which is defined as $\max _{\pi} \mathbb{E}_{\pi}(\mathbf{r}(s, a))$. The rightside inequality is by the definition of optimality.

$$
\mathbb{E}_{\pi_{*}^{\text {soft }}}(\mathbf{r}(s, a)) \leq \max _{\pi} \mathbb{E}_{\pi}(\mathbf{r}(s, a))=\mathbb{E}_{\pi_{*}}(\mathbf{r}(s, a)) .
$$

Before proving the leftside inequality, we first derive following inequality from Lemma 4 .

$$
V_{*} \leq V_{*}^{\text {soft }}
$$

where $*$ indicates an optimal solution. Then, the proof of the leftside inequality is done by using (14) as follows:

$$
\begin{aligned}
& \mathbb{E}_{\pi_{*}}(\mathbf{r}(s, a))=d^{\top} V_{*} \\
& \leq d^{\top} V_{*}^{\text {soft }}=J_{*}^{\text {soft }}=\mathbb{E}_{\pi_{*}^{\text {soft }}}(\mathbf{r}(s, a))+\alpha H\left(\pi_{*}^{\text {soft }}\right) \\
& \leq \mathbb{E}_{\pi_{*}^{\text {soft }}}(\mathbf{r}(s, a))+\frac{\alpha}{1-\gamma} \log (|\mathcal{A}|)(\because \text { Lemma } 5) .
\end{aligned}
$$

\section{APPENDIX B}

In this section, we present the full experimental results of reinforcement learning with a continuous action space. We performe experiments on Inverted Pendulum and Reacher and 28 algorithms are tested including our sparse exploration method and sparse Bellman update rule.

\section{REFERENCES}

[1] S. Brechtel, T. Gindele, and R. Dillmann, "Probabilistic decision-making under uncertainty for autonomous driving using continuous pomdps," in 17th International Conference on Intelligent Transportation Systems, October 2014, pp. 392-399.

[2] S. Ragi and E. K. P. Chong, "UAV path planning in a dynamic environment via partially observable markov decision process," IEEE Trans. Aerospace and Electronic Systems, vol. 49, no. 4, pp. 2397-2412, 2013.

[3] J. Hwangbo, I. Sa, R. Siegwart, and M. Hutter, "Control of a quadrotor with reinforcement learning," IEEE Robotics and Automation Letters, vol. 2, no. 4, pp. 2096-2103, 2017.

[4] J. Kober, J. A. Bagnell, and J. Peters, "Reinforcement learning in robotics: A survey," International Journal of Robotics Research, vol. 32, no. 11 , pp. 1238-1274, 2013.

[5] A. Y. Ng and S. J. Russell, "Algorithms for inverse reinforcement learning," in Proc. of the 7th International Conference on Machine Learning, June 2000, pp. 663-670.

[6] T. Haarnoja, H. Tang, P. Abbeel, and S. Levine, "Reinforcement learning with deep energy-based policies," in Proc. of the 34th International Conference on Machine Learning, August 2017, pp. 1352-1361.

[7] N. Heess, D. Silver, and Y. W. Teh, "Actor-critic reinforcement learning with energy-based policies," in Proc. of the Tenth European Workshop on Reinforcement Learning, June 2012, pp. 43-58.

[8] J. Schulman, P. Abbeel, and X. Chen, "Equivalence between policy gradients and soft q-learning," arXiv preprint arXiv:1704.06440, 2017.

[9] M. Tokic and G. Palm, "Value-difference based exploration: Adaptive control between epsilon-greedy and softmax," in KI 2011: Advances in Artificial Intelligence, 34th Annual German Conference on AI, October 2011, pp. 335-346.

[10] P. Vamplew, R. Dazeley, and C. Foale, "Softmax exploration strategies for multiobjective reinforcement learning," Neurocomputing, vol. 263, pp. 74-86, 2017.
[11] M. Bloem and N. Bambos, "Infinite time horizon maximum causal entropy inverse reinforcement learning," in 53rd IEEE Conference on Decision and Control, December 2014, pp. 4911-4916.

[12] C. Tsallis, "Possible generalization of boltzmann-gibbs statistics," Journal of statistical physics, vol. 52, no. 1, pp. 479-487, 1988.

[13] W. Wang and M. A. Carreira-Perpinán, "Projection onto the probability simplex: An efficient algorithm with a simple proof, and an application," arXiv preprint arXiv:1309.1541, 2013.

[14] D. R. Smart, Fixed point theorems. CUP Archive, 1980, vol. 66.

[15] T. P. Lillicrap, J. J. Hunt, A. Pritzel, N. Heess, T. Erez, Y. Tassa, D. Silver, and D. Wierstra, "Continuous control with deep reinforcement learning," arXiv preprint arXiv:1509.02971, 2015.

[16] J. Ye, "Constraint qualifications and necessary optimality conditions for optimization problems with variational inequality constraints," SIAM Journal on Optimization, vol. 10, no. 4, pp. 943-962, 2000.

[17] A. Martins and R. Astudillo, "From softmax to sparsemax: A sparse model of attention and multi-label classification," in International Conference on Machine Learning, June 2016, pp. 1614-1623.

[18] B. D. Ziebart, "Modeling purposeful adaptive behavior with the principle of maximum causal entropy," Ph.D. dissertation, Carnegie Mellon University, Pittsburgh, PA, USA, 2010.

[19] T. Schaul, J. Quan, I. Antonoglou, and D. Silver, "Prioritized experience replay," arXiv preprint arXiv:1511.05952, 2015.

[20] C. J. Watkins and P. Dayan, "Q-learning," Machine Learning, vol. 8, no. 3-4, pp. 279-292, 1992.

[21] H. van Hasselt, A. Guez, and D. Silver, "Deep reinforcement learning with double q-learning," in Proc. of the Thirtieth AAAI Conference on Artificial Intelligence, February 2016, pp. 2094-2100.

[22] E. Todorov, T. Erez, and Y. Tassa, "Mujoco: A physics engine for modelbased control," in International Conference on Intelligent Robots and Systems, October 2012, pp. 5026-5033. 


\begin{tabular}{|l|l|l|l|l|l|}
\hline The Number of Action & 3 & 101 & 1001 & 2001 & Average \\
\hline Sparse+SparseBellman-1 & 1000.0 & 996.8 & 1000.0 & 1000.0 & $\mathbf{9 9 9 . 2}$ \\
\hline Sparse+SparseBellman-0.1 & 1000.0 & 933.1 & 668.2 & 1000.0 & 900.3 \\
\hline Sparse+SparseBellman-0.01 & 1000.0 & 992.1 & 1000.0 & 1000.0 & $\mathbf{9 9 8 . 0}$ \\
\hline Sparse+SoftBellman-1 & 1000.0 & 1000.0 & 925.2 & 1000.0 & 981.3 \\
\hline Sparse+SoftBellman-0.1 & 1000.0 & 1000.0 & 1000.0 & 1000.0 & $\mathbf{1 0 0 0 . 0}$ \\
\hline Sparse+SoftBellman-0.01 & 782.7 & 988.6 & 775.8 & 1000.0 & 886.8 \\
\hline Sparse+Bellman-1 & 1000.0 & 1000.0 & 919.7 & 715.3 & 908.7 \\
\hline Sparse+Bellman-0.1 & 980.2 & 745.5 & 1000.0 & 1000.0 & 931.4 \\
\hline Sparse+Bellman-0.01 & 1000.0 & 1000.0 & 1000.0 & 1000.0 & $\mathbf{1 0 0 0 . 0}$ \\
\hline Soft+SparseBellman-1 & 673.9 & 835.5 & 53.3 & 1000.0 & 640.7 \\
\hline Soft+SparseBellman-0.1 & 688.0 & 1000.0 & 938.0 & 904.2 & 882.6 \\
\hline Soft+SparseBellman-0.01 & 993.0 & 1000.0 & 736.4 & 1000.0 & 932.3 \\
\hline Soft+SoftBellman-1 & 939.6 & 738.3 & 506.3 & 943.2 & 781.9 \\
\hline Soft+SoftBellman-0.1 & 1000.0 & 1000.0 & 1000.0 & 681.6 & 920.4 \\
\hline Soft+SoftBellman-0.01 & 1000.0 & 974.8 & 1000.0 & 1000.0 & 993.7 \\
\hline Soft+Bellman-1 & 668.9 & 621.5 & 668.7 & 643.2 & 650.6 \\
\hline Soft+Bellman-0.1 & 1000.0 & 1000.0 & 1000.0 & 1000.0 & $\mathbf{1 0 0 0 . 0}$ \\
\hline Soft+Bellman-0.01 & 977.6 & 1000.0 & 1000.0 & 1000.0 & 994.4 \\
\hline EpsGrdy+SparseBellman-1 & 479.8 & 669.0 & 344.5 & 678.1 & 542.9 \\
\hline EpsGrdy+SparseBellman-0.1 & 668.1 & 1000.0 & 351.1 & 666.6 & 671.4 \\
\hline EpsGrdy+SparseBellman-0.01 & 1000.0 & 124.6 & 477.5 & 667.8 & 567.5 \\
\hline EpsGrdy+SoftBellman-1 & 940.3 & 684.9 & 658.3 & 505.6 & 697.3 \\
\hline EpsGrdy+SoftBellman-0.1 & 338.5 & 376.8 & 1000.0 & 1000.0 & 678.8 \\
\hline EpsGrdy+SoftBellman-0.01 & 551.5 & 652.8 & 735.2 & 677.9 & 654.3 \\
\hline EpsGrdy+Bellman-1 & 332.7 & 1000.0 & 1000.0 & 369.8 & 675.6 \\
\hline EpsGrdy+Bellman-0.1 & 1000.0 & 618.7 & 1000.0 & 771.7 & 847.6 \\
\hline EpsGrdy+Bellman-0.01 & 462.6 & 676.5 & 698.0 & 48.1 & 471.3 \\
\hline DDPG & 253.1 & & & & 253.1 \\
\hline
\end{tabular}

TABLE II: Expected return of Inverted Pendulum. Top five performances are marked in bold.

\begin{tabular}{|l|l|l|l|l|}
\hline The Number of Action & 3 & 101 & 1001 & 2001 \\
\hline Sparse+SparseBellman-1 & 1164 & 692 & 742 & 864 \\
\hline Sparse+SparseBellman-0.1 & 1060 & 2923 & 3998 & 599 \\
\hline Sparse+SparseBellman-0.01 & 685 & 1431 & 1010 & 811 \\
\hline Sparse+SoftBellman-1 & 863 & 1316 & 1698 & 657 \\
\hline Sparse+SoftBellman-0.1 & 914 & 901 & 857 & 802 \\
\hline Sparse+SoftBellman-0.01 & 3342 & 907 & 3930 & 522 \\
\hline Sparse+Bellman-1 & 879 & 668 & 2337 & 3137 \\
\hline Sparse+Bellman-0.1 & 937 & 3925 & 773 & 1030 \\
\hline Sparse+Bellman-0.01 & 999 & 329 & 962 & 962 \\
\hline Soft+SparseBellman-1 & 3789 & 3416 & 3996 & 2684 \\
\hline Soft+SparseBellman-0.1 & 3844 & 2835 & 1494 & 2771 \\
\hline Soft+SparseBellman-0.01 & 854 & 545 & 3814 & 999 \\
\hline Soft+SoftBellman-1 & 1885 & 3666 & 3994 & 3912 \\
\hline Soft+SoftBellman-0.1 & 869 & 780 & 787 & 3871 \\
\hline Soft+SoftBellman-0.01 & 533 & 1241 & 2565 & 3020 \\
\hline Soft+Bellman-1 & 3898 & 3947 & 3978 & 3758 \\
\hline Soft+Bellman-0.1 & 876 & 1923 & 954 & 807 \\
\hline Soft+Bellman-0.01 & 1419 & 689 & 755 & 1265 \\
\hline EpsGrdy+SparseBellman-1 & 3978 & 3993 & 4000 & 3863 \\
\hline EpsGrdy+SparseBellman-0.1 & 3895 & 2449 & 4000 & 3910 \\
\hline EpsGrdy+SparseBellman-0.01 & 3437 & 4000 & 3962 & 3777 \\
\hline EpsGrdy+SoftBellman-1 & 2959 & 3919 & 3715 & 4000 \\
\hline EpsGrdy+SoftBellman-0.1 & 3997 & 3969 & 3037 & 2509 \\
\hline EpsGrdy+SoftBellman-0.01 & 3976 & 3936 & 3785 & 3784 \\
\hline EpsGrdy+Bellman-1 & 4000 & 2603 & 1093 & 3969 \\
\hline EpsGrdy+Bellman-0.1 & 2584 & 3897 & 3160 & 3846 \\
\hline EpsGrdy+Bellman-0.01 & 3891 & 3699 & 3905 & 3993 \\
\hline
\end{tabular}

TABLE III: The number of episodes required to reach the threshold return, 980. 


\begin{tabular}{|l|l|l|l|l|l|}
\hline The Number of Action & 9 & 121 & 961 & 2601 & Average \\
\hline Sparse+SparseBellman-1 & -7.7 & -7.8 & -10.1 & -11.5 & -9.3 \\
\hline Sparse+SparseBellman-0.1 & -11.3 & -5.7 & -5.4 & -5.5 & -7.0 \\
\hline Sparse+SparseBellman-0.01 & -11.3 & -8.7 & -8.6 & -6.3 & -8.7 \\
\hline Sparse+SoftBellman-1 & -7.6 & -10.5 & -11.5 & -10.0 & -9.9 \\
\hline Sparse+SoftBellman-0.1 & -10.4 & -5.8 & -5.5 & -9.3 & -7.8 \\
\hline Sparse+SoftBellman-0.01 & -11.2 & -6.4 & -8.9 & -6.4 & -8.2 \\
\hline Sparse+Bellman-1 & -7.6 & -7.7 & -5.7 & -10.2 & -7.8 \\
\hline Sparse+Bellman-0.1 & -10.8 & -5.5 & -5.4 & -5.8 & $-\mathbf{6 . 9}$ \\
\hline Sparse+Bellman-0.01 & -11.6 & -5.9 & -5.9 & -9.4 & -8.2 \\
\hline Soft+SparseBellman-1 & -52.0 & -48.0 & -29.6 & -39.3 & -42.2 \\
\hline Soft+SparseBellman-0.1 & -7.4 & -22.4 & -20.8 & -25.5 & -19.0 \\
\hline Soft+SparseBellman-0.01 & -11.1 & -5.5 & -5.5 & -9.2 & -7.8 \\
\hline Soft+SoftBellman-1 & -52.2 & -43.1 & -46.8 & -44.1 & -46.5 \\
\hline Soft+SoftBellman-0.1 & -7.5 & -22.5 & -23.9 & -32.9 & -21.7 \\
\hline Soft+SoftBellman-0.01 & -11.6 & -5.7 & -5.5 & -7.6 & -7.6 \\
\hline Soft+Bellman-1 & -51.4 & -51.7 & -44.2 & -41.2 & -47.1 \\
\hline Soft+Bellman-0.1 & -7.1 & -10.0 & -26.7 & -27.5 & -17.8 \\
\hline Soft+Bellman-0.01 & -11.3 & -5.3 & -5.3 & -10.2 & -8.0 \\
\hline EpsGrdy+SparseBellman-1 & -11.2 & -7.6 & -5.6 & -6.2 & -7.6 \\
\hline EpsGrdy+SparseBellman-0.1 & -11.2 & -5.9 & -5.8 & -6.1 & -7.2 \\
\hline EpsGrdy+SparseBellman-0.01 & -10.9 & -5.9 & -5.5 & -6.0 & -7.1 \\
\hline EpsGrdy+SoftBellman-1 & -10.5 & -5.9 & -5.7 & -6.1 & -7.0 \\
\hline EpsGrdy+SoftBellman-0.1 & -11.3 & -5.7 & -5.6 & -6.2 & -7.2 \\
\hline EpsGrdy+SoftBellman-0.01 & -10.8 & -6.2 & -12.1 & -9.5 & -9.6 \\
\hline EpsGrdy+Bellman & -10.8 & -6.5 & -5.7 & -6.5 & -7.4 \\
\hline EpsGrdy+Bellman & -11.1 & -6.2 & -5.9 & -5.9 & -7.3 \\
\hline EpsGrdy+Bellman & -10.6 & -9.4 & -8.4 & -6.5 & -8.7 \\
\hline DDPG & -10.1 & & & & -10.1 \\
\hline & & & & & \\
\hline
\end{tabular}

TABLE IV: Expected return of Reacher. Top five performances are marked in bold.

\begin{tabular}{|l|l|l|l|l|}
\hline The Number of Action & 9 & 121 & 961 & 2601 \\
\hline Sparse+SparseBellman-1 & 9193 & 9648 & 7275 & 9065 \\
\hline Sparse+SparseBellman-0.1 & 9791 & 5837 & 5779 & 6851 \\
\hline Sparse+SparseBellman-0.01 & 9783 & 6456 & 6631 & 7941 \\
\hline Sparse+SoftBellman-1 & 9126 & 9834 & 7603 & 8503 \\
\hline Sparse+SoftBellman-0.1 & 9779 & 5449 & 5642 & 7509 \\
\hline Sparse+SoftBellman-0.01 & 9795 & 5011 & 7260 & 7768 \\
\hline Sparse+Bellman-1 & 9073 & 9619 & 5646 & 8371 \\
\hline Sparse+Bellman-0.1 & 9756 & 5366 & 5338 & 6936 \\
\hline Sparse+Bellman-0.01 & 9797 & 5204 & 6525 & 7965 \\
\hline Soft+SparseBellman-1 & 10000 & 10000 & 10000 & 10000 \\
\hline Soft+SparseBellman-0.1 & 8801 & 9998 & 10000 & 10000 \\
\hline Soft+SparseBellman-0.01 & 9783 & 4988 & 5934 & 8774 \\
\hline Soft+SoftBellman-1 & 10000 & 10000 & 10000 & 10000 \\
\hline Soft+SoftBellman-0.1 & 8810 & 9999 & 10000 & 10000 \\
\hline Soft+SoftBellman-0.01 & 9794 & 4597 & 5927 & 7915 \\
\hline Soft+Bellman-1 & 10000 & 10000 & 10000 & 10000 \\
\hline Soft+Bellman-0.1 & 8700 & 9999 & 10000 & 10000 \\
\hline Soft+Bellman-0.01 & 9790 & 4810 & 6004 & 8737 \\
\hline EpsGrdy+SparseBellman-1 & 9861 & 6909 & 6994 & 7977 \\
\hline EpsGrdy+SparseBellman-0.1 & 9850 & 6808 & 6775 & 7873 \\
\hline EpsGrdy+SparseBellman-0.01 & 9847 & 7079 & 6850 & 7923 \\
\hline EpsGrdy+SoftBellman-1 & 9850 & 6839 & 6858 & 8026 \\
\hline EpsGrdy+SoftBellman-0.1 & 9844 & 6918 & 6752 & 7849 \\
\hline EpsGrdy+SoftBellman-0.01 & 9841 & 7176 & 9803 & 8114 \\
\hline EpsGrdy+Bellman & 9842 & 6797 & 6933 & 8001 \\
\hline EpsGrdy+Bellman & 9846 & 6680 & 7051 & 7845 \\
\hline EpsGrdy+Bellman & 9864 & 7192 & 6928 & 7925 \\
\hline
\end{tabular}

TABLE V: The number of episodes required to reach the threshold return, -6 . 